\title{
Mesenchymal stem cell-derived exosomal microRNA-34c-5p ameliorates renal pericyte activation by inhibiting core fucosylation
}

Hu Xuemei

the First Affiliated Hospital, Dalian Medical University

Nan Shen

the First Affiliated Hospital, Dalian Medical University

Anqi Liu

the First Affiliated Hospital, Dalian Medical University

Weidong Wang

First Affiliated Hospital of Dalian Medical University

Lihua Zhang

Dalian Institute of Chemical Physics

\section{Zhigang Sui}

National Chromatographic Research and Analysis Center, Key Lab of Separation Sciences for Analytical

Chemistry, Dalian Institute of Chemical Physics, Chinese Academy of Sciences, Dalian, China

Qingzhu Tang

the First Affiliated Hospital, Dalian Medical University

Xiangning Du

the First Affiliated Hospital, Dalian Medical University

Ning Yang

the First Affiliated Hospital, Dalian Medical University

Wantao Ying

State Key Laboratory of Proteomics, Beijing Proteome Research Center, National Center for Protein

Sciences

\section{Biaojie Qin}

the First Affiliated Hospital, Dalian Medical University

\section{Zhitong Li}

the First Affiliated Hospital, Dalian Medical University

Lin Li

the First Affiliated Hospital, Dalian Medical University

\section{Nan Wang}

the First Affiliated Hospital, Dalian Medical University

Hong-Li Lin ( $\sim$ linhongli@vip.163.com ) 


\section{Article}

Keywords: mesenchymal stem cells, exosomes, pericyte, core fucosylation, renal interstitial fibrosis, microRNA-34c-5p

Posted Date: November 13th, 2020

DOI: https://doi.org/10.21203/rs.3.rs-88403/v1

License: (c) (1) This work is licensed under a Creative Commons Attribution 4.0 International License. Read Full License 


\section{Abstract}

Renal interstitial fibrosis (RIF) is an incurable pathological lesion in progressive chronic kidney diseases. Myofibroblast proliferation and microvascular damage are two important events in RIF, and pericytes are a major source of myofibroblasts in the kidney. However, the underlying mechanisms remain poorly characterized. We report that core fucosylation (CF), a post-translational modification of proteins, is essential for pericyte activation by regulating profibrotic and antifibrotic signaling pathways as a "hublike" target. Mesenchymal stem cell (MSC)-derived exosomes reside specifically in the obstructed kidney and deliver microRNA (miR)-34c-5p to inhibit CF, reducing pericyte activation and renal fibrosis. Furthermore, we clarify that the CD81-EGFR ligand-receptor complex aids the entry of exosomal miR$34 c-5 p$ into pericytes. Our results reveal a novel mechanism of pericyte activation based on CF and suggest a potential use of MSC exosomes as a new therapeutic strategy for RIF by inhibiting CF, the hublike target of profibrotic signaling activation.

\section{Introduction}

Persistent and unremitting renal interstitial fibrosis (RIF) ultimately leads to end-stage renal diseases, which are fatal diseases that force patients to undergo dialysis for life or to undergo kidney transplantation ${ }^{1}$. Unfortunately, there is no effective method for halting RIF to date. A progressive increase in myofibroblasts in the renal interstitium is well recognized as the main culprit in RIF $^{2}$. Meanwhile, the destruction of renal interstitial microvessels during RIF has recently become a matter of increasing concern ${ }^{3}$.

Pericytes are perivascular cells attached to the abluminal surface of the renal interstitial microvessels responsible for stabilizing vascular walls and other important physiological functions. Upon activation, pericytes detach from the vascular basement membrane, and are prone to instability, ultimately leading to rarefaction in $\mathrm{RIF}^{4}$

Importantly, Duffield and LeBleu have shown that pericyte-myofibroblast transition is the major source of myofibroblasts in RIF, which suggests that pericytes can be identified as a culprit obviously involved in both the microvessel rarefaction and myofibroblast proliferation in $\mathrm{RIF}^{2,5}$. Accordingly, the inhibition of pericyte activation should be exploited for developing a novel and effective therapeutic against RIF ${ }^{6}$.

The activation of multiple cross-linked fibrogenic signaling pathways is the basis for pericyte activation ${ }^{7}$ 8, 9. Therefore, the novel "hub-like" target of these signaling pathways might be a candidate point for suppressing the overactivation simultaneously. As post-translational modification of proteins is essential for their functions, it would be more reasonable to target a common modification site of the pathway proteins regulating signaling pathway activity rather than interfering with the protein molecules individually to achieve the aim of regulating signaling pathway activity ${ }^{10}$. We have verified that core fucosylation (CF), which is specifically catalyzed by a1,6-fucosyltransferase (FUT8), is a key posttranslational modification of glycoproteins, and can simultaneously regulate both the TGF- $\beta$-SMAD and 
PDGF-ERK signaling pathways during pericyte activation and $\mathrm{RIF}^{6,7,11,12}$. These findings suggest that CF might be a promising therapeutic hub-like target.

Cell-based therapies that involve mesenchymal stem cells (MSCs) have emerged as promising options for kidney diseases ${ }^{13,14,15}$. Although MSCs have beneficial effects against RIF, their clinical application faces many challenges, including ethics, safety, tumorigenesis, immunosuppression, and multidirectional differentiation ${ }^{16}$. Therefore, we used MSC-derived exosomes instead of MSCs as a candidate regulator of CF.

Here, we present evidence that MSC-derived exosomes reside specifically in obstructed kidney, and then deliver microRNA (miR)-34c-5p into pericytes, assisted by the CD81-EGFR complex located on the pericyte membrane, to inhibit the CF of several important profibrotic proteins and thereby ameliorate pericyte activation and renal fibrosis.

\section{Results}

MSCs inhibited CF during pericyte activation.CF was elevated in pericyte activation and RIF(Supplementary information 1).To investigate whether MSCs can inhibit CF during pericyte activation, we co-cultured activated pericytes with MSCs in Transwell chambers in vitro. We found that MSCs markedly suppressed both the increase in FUT8 protein expression and FUT8 activity, downregulated CF (Fig. 1c-e), and significantly decreased a-SMA expression and pericyte transition into spindle myofibroblasts (Fig. 1a,b). These findings suggest that MSCs inhibit CF levels and decrease pericyte activation.

Nest, we assessed whether MSCs inhibit CF in the UUO kidney during renal fibrosis. MSCs $\left(1 \times 10^{6}\right)$ were injectedinto mouse tail vein immediately after UUO operation. The MSCs markedly inhibited CF (Fig. 1h) and markedly attenuated bothpericyte detachment from endothelial cells and pericyte transition to myofibroblasts at 7 days after UUO (Fig. 1f,g). We also detected thedistribution of MSCs in the mice by injecting MSC/RFP into PDGFRß-EGFP fluorescent reporter mice via the tail vein (Supplementary information 2). MSCs were not seen in UUO mouse kidneys. These data suggest that the benefit of MSCs against renal fibrosis in UUO mice might follow the paracrine mode rather than having direct or contact effects.

MSC-derived exosomes ameliorated pericyte activation and renal fibrosis by inhibiting CF. An increasing number of studies have indicated that the therapeutic effects of MSCs are largely mediated by paracrine factors, including exosomes ${ }^{17,18}$. Accordingly, we investigated whether the therapeutic effects result from MSC-derived exosomes. Exosomes were collected from the supernatant of MSCs cultured in serum-free medium and were identified by electron microscopy, nanoparticle tracking analysis, and western blotting (Fig. 3a). First, we performed a tracer experiment to detect exosome distribution. The luciferase marker illustrated the PKH67 fluorescence-labeled exosomes; exosome secretion peaked at $24 \mathrm{~h}$ (Fig. 2a). In vitro imaging of MSCs co-cultured with TGF- $\beta 1$-stimulated pericytes in Transwell chambers showed that the 
fluorescently labeled exosomes were visible in the pericytes; exosome distribution in the pericytes peaked at $48 \mathrm{~h}$ (Fig. 2b). Furthermore, exosome distribution after injection into UUO mouse tail vein was traced using an in vivo imaging system. We found that exosome targeting accumulated dramatically in the obstructed kidney 3 days after UUO, and peaked at 7 days post-UUO. Besides, a few exosomes were observed in the heart, spleen, liver, lung, and brain, with no obvious pathological changes (Fig. 2C, d). These results indicate that exosomes accumulate mainly in the obstructed kidney.

We then prepared MSC CM, exosomes extracted from CM (Exo), and exosome-free MSC CM [CM(-)Exo], and tested their effects on CF and pericyte activation in cultured pericytes and in UUO mice. Notably, MSCs, exosomes, and CM all suppressed FUT8 protein overexpression andactivity effectively and synchronously with LCA elevation in the pericytes following 48-h incubation with TGF- $\beta 1$ (Fig. 3d-f). Incubation with MSCs, exosomes, and CM alleviated the number of pericytes transitioning to myofibroblasts. However, CM(-)Exo did not exhibit the above inhibitory effects (Fig. $\mathbf{3 b}, \mathbf{c}$ ).

We then investigated the effects of exosomes on UUO mice. LCA levels were decreased following the injection of exosomes, CM, and MSCs (Fig. 3i). The degree of pericyte separation from vascular endothelial cells and a-SMA expression levels were decreased, indicating that pericyte-myofibroblast transition was inhibited by exosomes, MSCs, and CM, but not by CM(-)Exo (Fig. 3h). CM and exosome injection alleviated RIF, an effect similar to that of MSCs, but CM(-)Exo had no such effects (Fig. 3g). These findings suggest that MSCs inhibit pericyte activation through exosomes in UUO mice. However, whether the role of MSCs and exosomes is mainly dependent on CF inhibition is not known.

Further, we performed a FUT8 rescue experiment. RT-PCR, immunofluorescence, and western blotting were used to verify that the FUT8 plasmid was successfully transfected into the pericytes (Fig. 4a). Following the transfection of FUT8, FUT8 and LCA expression was increased, the degree of pericyte-myofibroblast transition was aggravated, and the curative effect of MSCs and exosomes was significantly reversed (Fig. $\mathbf{4 b}-\mathbf{f}$ ). These results reveal that the novel mechanism of exosome inhibition of pericyte activation and renal fibrosis occurs mainly via downregulating $\mathrm{CF}$.

Exosome-induced inhibition of CF inactivated multiple signaling pathways.Previously, we found that $\mathrm{CF}$ regulates the activity of both the TGF- $\beta-$ SMAD and PDGF-ERK pathways in pericyte activation and in $\mathrm{RIF}^{6}$. We hypothesized that the inhibitory effects of the exosomes on CFmay inactivate multiple profibrotic pathways simultaneously. As the TGF- $\beta$-SMAD2/3, PDGF-ERK1/2, and EGFR-ERBB3 pathways have been recognized as key classical profibrotic signaling pathways in renal pericytes ${ }^{6,19}$, and the BMP7-SMAD6/7, WNT5a-PCP, and NOTCH3-JAGGED pathways have been recognized as antifibrotic signaling pathways in renal fibrosis ${ }^{20,21,22}$, we investigated the effect of the exosomes on these pathways. Lectin blotting and immunofluorescence analysis showed that the addition of exosomes decreased the CF of TGF $\beta$ R, PDGFR 3 , EGFR, WNT5a, and NOTCH3 in the pericytes following 48-h incubation with TGF- $\beta 1$. Interestingly, the exosomes had no effect on the expression levels of the above membrane proteins. In addition, the exosomes inhibited the phosphorylation of SMAD2/3, ERK1/2, and ERBB3 without affecting protein expression levels. Furthermore, we detected the role of MSCs, CM, and 
exosomes in the BMP-SMAD6/7 signaling pathway, a typical antifibrotic signaling pathway that competitively inhibits SMAD2/3. We found that CF did not modify the BMP7 receptor, and observed an opposite change for BMP-SMAD6/7 compared to TGF- $\beta$-SMAD2/3. However, the addition of exosomefree $\mathrm{CM}$ did not have any of the stimulatory or inhibitory effects defined above. CF modified the EGFR, WNT5a, and NOTCH3 receptors, and we observed the same changes for ERBB3, PCP, and JAGGED as compared to TGF- $\beta$-SMAD2/3 ${ }^{23}$. However, the addition of exosome-free CM did not have any of the stimulatory or inhibitory effects defined above (Fig. 5a-e). Our study is the first to show that the beneficial effects of exosomes on renoprotection are due to decreased CF, which leads to both the suppression of several important fibrogenic pathways and the activation of anti-fibrogenic signaling pathways.

\section{Exosomes downregulated $\mathrm{CF}$ by delivering miR-34c-5p during pericyte activation and renal fibrosis.MSC-} derived exosomes perform biological functions by releasing miRNAs ${ }^{24,25,26}$. Therefore, we screened the miRNA responsible for regulating FUT8 activity. MSCs were cultured for $0 \mathrm{~h}, 24 \mathrm{~h}$, and $48 \mathrm{~h}$ without serum, the $\mathrm{CM}$ was collected, and the exosomes were isolated for miRNA omics analysis. A total of $176 \mathrm{miRNAs}$ with different expression changes at the three timepoints weredetected. These miRNAs overlapped with 71 miRNAs from the miRDB miRNA library that specifically regulate FUT8, and only a single miRNA, i.e., miR-34c-5p, was identified. The heatmap showed significant differential expression of miR-34c-5p in the $24 \mathrm{~h}$ group compared with the $0 \mathrm{~h}$ group (Fig. 6a). Further, we verified whether FUT8 is regulated by miR$34 c-5 p$ using the luciferase assay. The data indicated that miR-34c-5p markedly reduced the luciferase activity of the wild-type (WT) FUT8 reporter plasmid as compared to the negative control (NC) (Fig. 6b), whichconfirmed that miR-34c-5p could specifically interact and regulate with FUT8.

To examine the mechanism of miR-34c-5p regulation of FUT8 during pericyte activation and renal fibrosis, miR-34c-5p mimics and inhibitor encoding GFP were commercially constructed (GenePharma). The synthetic mimic or inhibitor was transfected into MSCs, and the exosomes were collected from the supernatant. RT-PCR and flow cytometry showed that FUT8 was dramatically reduced and increased after miR-34c-5p mimic and inhibitor transfection, respectively, in the MSC and exosome groups

(Supplementary Fig. 3a). These data indicate the successful generation of miR-34c-5p mimic and inhibitor. Besides exosomes and MSCs, the miR-34c-5p mimic greatly decreased pericyte detachment and pericyte transition to myofibroblasts following 48-h incubation with TGF- $\beta 1$, and downregulated a-SMA expression and CF (Fig. 6c). The inhibitory effect of miR-34c-5p in the UUO mouse kidney during RIF was then assessed. Besides injection of exosomes and MSCs, miR-34c-5p mimic markedly decreased tubulointerstitial damage and fibrosis at 7 days after UUO, and downregulated a-SMA expression and CF; miR-34c-5p was also observed in other organs after injection with the miR-34c-5p mimic (Fig. 6d, Supplementary Fig. 3c). The data show that the inhibitory effect of exosomes on CF and pericyte activation is mainly dependent on miR-34c-5p. We performed a tracer experiment to elucidate the main methods of miR-34c-5p protection. Fluorescent fam-labeled miR-34c-5pwas transfected into MSCs, and the fluorescence-labeled miR-34c-5p in the CM was illustrated using the luciferase marker. TGF- $\beta 1$ stimulated pericytes were cultured with the $\mathrm{CM}$ in Transwell chambers, and the CM was injected into the 
mice via the tail vein. The fluorescence-labeled miR-34c-5pwas observed in the pericytes and UUO mouse kidney (Supplementary Fig. 3b).

CD81-EGFR complex formation aided miR-34c-5p entry into pericytes to downregulate CF.Finally, we explored how miR-34c-5p is delivered from exosomes into pericytes to inhibit CF. Recent discoveries have highlighted the fact that exosomes mediate crosstalk between different cell types by miRNA delivery to specific recipient cell types via receptor-mediated binding 27,28 . Here, we screened out 1708 pericyte membrane proteins and 1687 exosome membrane proteins using proteomic analysis. Eighty-four pericyte-exosome interacting membrane proteins were identified by examining protein-protein interaction using STRING software. A protein-protein interaction network was drawn to provide a framework for visualizing receptor-mediated binding. As shown in the network, larger nodesindicated more protein interactions, suggesting that the protein is more important in the network. The nodes was largest for EGFR protein. Among the proteins closest to EGFR, the nodes was largest for CD81. We hypothesized that the EGFR-CD81 complex might play an important role in mediating miR-34c-5p delivery from exosomes to pericytes (Fig. 7a).

Then, we constructed the spatial 3D structures of a full-size EGFR and CD81 using computer-based techniques. Interactions between EGFR and the CD81 extracellular domain estimated using a molecular docking approach aided the identification of three sites for EGFR binding to CD81. The CD81 extracellular region binds to the EGFR ligand-binding region to form a complex,and FUT8 does not bind in the EGFR ligand-binding region and does not affect CD81-EGFR complex formation (Fig. 7b, Supplementary Fig. 4a). Collectively, these data provide evidence for the stable coupling of EGFR with CD81. Immunofluorescence staining aimed at verifying CD81-EGFR complex formation showed decreased EGFR fluorescence after exosome treatment. CD81 binds to the EGFR ligand-binding region mainly by hydrogen bonds and hydrophobic action, affecting the conformation of amino acid residues and leading to the decreased EGFR fluorescence intensity. The 3D fluorescence showed the binding of exogenous CD81 to EGFR (Fig. 7c).

To study the function of the CD81-EGFR complex in the process of pericyte activation and renal fibrosis, GFP-encoding CD81 and EGFR shRNA recombinant adenovirus vectors werecommercially constructed (GenePharma). Adenoviral transfections were performed on MSCs, pericytes, and mice through tail vein injection. RT-PCR and flow cytometry showed that CD81 and EGFR were dramatically reduced after CD81 shRNA and EGFR shRNA transfection (Supplementary Fig. 4b, c). These data provide evidence for the stable coupling between EGFR and CD81.

We also used rhodamine-labeled LCA (LCA-TRITC) to investigate whether the CD81-EGFR complex aids miR-34c-5p entry into pericytes to inhibit CF. Following the inhibition of CD81 or EGFR, exosomes could not inhibit CF or pericyte activation, and we could not detect miR-34c-5p in the pericytes orkidney (Fig. 7d). The data show that CD81-EGFR complex formation aids miR-34c-5p entry into pericytes.

\section{Discussion}


MSCs can repair kidney injury effectively and reduce RIF progression $14,17,29,30,31,32,33$, but the mechanism warrants further exploration. In the present study, we found that MSCs inhibit both CF and pericyte activation, while FUT8 overexpression disabled the efficacy of the MSCs. The data demonstrate that MSCs reduced pericyte activation and RIF mainly by regulating CF. To the best of our knowledge, this is the first report that MSCs can antagonize pericyte activation by regulating post-translational modification of proteins.

MSCs function through local colonization or in a paracrine manner ${ }^{34}$. However, local colonization is in the minority ${ }^{32}$. Previously, we found that although MSCs injected through mouse tail vein decreased kidney injury, the MSCs mainly accumulated in the lungs instead of the injured kidney ${ }^{35}$. Our data show that MSCs in the upper Transwell chamber reduced pericyte activation in the bottom chamber with no contact, and exogenous red fluorescent-labeled MSCs did not accumulate in the obstructed kidney. We deduced that the benefits of MSCs were not due to colonization but mainly through a paracrine manner.

MSC exosome secretion can contribute greatly to the effect of MSCs, such as regulating the development of individual organisms, cell activation, and repairing damaged tissues ${ }^{18,36,37,38,39}$. Previously, we explored a new method for exosome enrichment based on polyethylene glycol(PEG) secondary precipitation, which was simple and efficient and did not affect exosome activity ${ }^{40}$. In this study, the dynamic tracer for fluorescent exosomes demonstrated that, at 1,3, and 7 days after tail vein injection, exosomes mainly accumulated in the obstructed kidney, while that in the contralateral kidney were barely detectable. As predicted, obvious exosome florescence signals were detected in renal tubular cells (data not shown), which is similar to the findings in acute kidney injury ${ }^{41,42}$. Here, we also monitored other organs, i.e., the heart, liver, spleen, lung, brain, and observed no pathological change when the exosomes were injected by vail vein injection. These results suggest that exosomes may be desirable therapeutic tools with good targeting ability, acceptable durability, and safety. MSCs may be recruited by receptormediated interaction ${ }^{43}$. Exosomes bear the same membrane receptors as MSCs. It is possible that exosomes may accumulate at the site of injury by similar mechanisms. However, the specific colonization mechanism and long-term efficacy require further investigation.

Our data show that CM, exosomes, and MSCs have similar inhibitory effects on pericyte activation and $\mathrm{CF}$, while exosome-free $\mathrm{CM}$ was ineffective both in vitro and in vivo. FUT8 overexpression disabled the efficacy of the exosomes. The data demonstrate that exosomes reduce pericyte activation and RIF mainly by regulating $\mathrm{CF}$.

Interestingly, we found that the therapeutic effect of MSCs was slightly better than that of exosomes, but was almost equivalent to that of $\mathrm{CM}$. We postulated that there were other cytokines in the $\mathrm{CM}$, and exosomes were virtually the main therapeutic component of CM. The MSCs exerted inhibitory effects mainly through exosome secretion. What is worth considering is how the effect of exosomes freed from MSCs can be enhanced. 
The activation of multiple signaling pathways is the initiative basis of pericyte activation and RIF. Regarding the important fibrogenic signaling pathways, we investigated the EGFR-ERBB3, TGF $\beta$ RSMAD2/3, and PDGFR-ERK1/2 pathways. The MSCs and exosomes did not affect the expression of membrane receptors in these signaling pathways, while both membrane receptor $\mathrm{CF}$ and phosphorylation of the downstream response elements were decreased remarkably. This inhibitory regulation of the fibrogenic signaling pathways may account for the therapeutic benefit of MSCs and exosomes for pericyte activation and RIF. Several studies have reported the inefficiency of single pathway blockage in mitigating fibrosis ${ }^{6,7,9}$. The compensation of other fibrogenic pathways or the relative inadequacy of anti-fibrosis pathways might account for the inefficiency of single pathway blockage. Then, we investigated the reported anti-fibrogenic signaling pathways, i.e., NOTCH3-PCP and WNT5A-JAGGED. Surprisingly, the exosomes greatly decreased both downstream response element expression and membrane receptor CF, while BMP7 expression (not regulated by CF) was not affected by TGF- $\beta 1$ or the exosomes. Its downstream SMAD6/7 demonstrated the opposite change to P-SMAD2/3; theoretically, the anti-fibrogenic function of BMP7-SMAD6/7 remained unaffected ${ }^{44}$. These data suggest that exosomes have inhibitory effects specifically for CF-regulated signaling pathways. Intervention for the hub-like control of multiple signaling pathways, such as CF in the present study, may be a more effective and specific strategy for inhibiting cellular activation and organ fibrosis.

MSC-derived exosomes are extracellular vesicles and can deliver functionally active materials such as proteins, miRNAs, and non-coding RNAs through membrane ligand-receptor interactions $24,27,45,46,47,48$. Exosomes can inhibit renal tubular epithelial cells and endothelial cell damage to play a role in renal tissue injury repair via miRNAs ${ }^{24,45}$; however, it has not been reported in pericytes. Therefore, we speculated that some miRNAs contained in exosomes might be involved in pericyte activation. We used exosome transcriptome and miRNA library regulation of FUT8 to screen out miR-34c-5p. miR-34c-5p reduces inflammation and fibrosis ${ }^{48}$, but the mechanism is unclear. Accordingly, we used a fluorescent reporter enzyme reaction to verify that FUT8 is a substrate of miR-34c-5p. We confirmed that exosomes regulate $\mathrm{CF}$ to inhibit pericyte activation and RIF mainly by delivering miR-34c-5p from MSCs to pericytes. However, the specific mechanism of miR-34c-5p delivery is unclear.

There are two types of exosome signaling: membrane fusion and endocytosis. Ligands-receptors form an endocytic complex to aid miRNA entry into recipient cells ${ }^{27,28,36}$. Here, exosome and pericyte proteomics screened out the membrane ligand and target receptor: CD81-EGFR. CD81 is a member of a four-transmembrane protein that binds to fusion receptor cells ${ }^{49}$. Here, we used computer simulation, 3D fluorescence imaging, and blockage experiments to confirm that exosomes delivered miR-34c-5p via CD81-EGFR complexes to regulate CF and pericyte activation in RIF. In summary, MSC-derived exosomes deliver miR-34c-5p into pericytes through the CD81-EGFR endocytic complex to downregulate CF, hence inhibiting CF-regulated multiple signaling pathways and ameliorating pericyte activation and RIF. From the point of glycolysation,we reveal a novel therapeutic mechanism of mesenchymal stem cells on renal interstitial fibrosis, which provide a basis for clinical application. 


\section{Methods}

MSC culture. C57BL/6 mouse bone marrow-derived MSCs/red fluorescent protein (RFP) were obtained commercially (Cyagen Biosciences, Sunnyvale, CA, USA). The culture was initiated following the manufacturer's instructions. The MSCs were placed in $25 \mathrm{~cm}^{2}$ culture flasks and cultured in MSC growth medium (Cyagen Biosciences) at $37^{\circ} \mathrm{C}$ under $5 \% \mathrm{CO}_{2}$ and $90 \%$ humidity. The medium was changed every 3 days. Passage 6-8 MSCs were used for the experiments. MSC/RFP identification was confirmed commercially (Cyagen Biosciences). Following previously described methods ${ }^{33}$, fluorescence-activated cell sorting (Beckman Coulter, Indianapolis, IN, USA) was used to examine the representative markers of MSCs [CD45, CD90 (BD Biosciences, San Diego, CA, USA), CD11a, CD54 (AbD Serotec, Oxford, UK)], and MSC multilineage differentiation was examined under adipogenic and osteogenic differentiation conditions.

Preparation of MSC exosomes, conditioned medium (CM), and exosome-free CM. MSCs cultured for 24-h in serum-free medium were removed from the incubator and observed under a microscope. The supernatant was collected and centrifuged ( $500 \times g, 20 \mathrm{~min}$ ). After centrifugation, the supernatant was placed in an ultrafiltration tube and centrifuged $(5000 \times g, 100 \mathrm{~min})$ to condense the culture volume by 30 times to prepare $\mathrm{CM}$ for injection, which was then aliquoted and stored at a density of $1 \times 10^{6}$ cells. The same ultracentrifugation method was used for producing the exosome-free CM: MSC supernatant medium collected for $24 \mathrm{~h}$ was centrifuged in a centrifuge tube $(500 \times \mathrm{g}, 20 \mathrm{~min})$; after centrifugation, the supernatant was placed in a new centrifuge tube and centrifuged again $(10,000 \times g, 20 \mathrm{~min})$. The supernatant was then centrifuged again in an ultracentrifuge tube $(100,000 \times g, 180 \mathrm{~min})$. The supernatants were collected as exosome-free $\mathrm{CM}$. The pellet in the ultracentrifuge tube was resuspended in phosphate-buffered saline (PBS) and transferred to a new ultracentrifuge tube $(100,000 \times g, 180 \mathrm{~min})$. The supernatant was discarded and the pelleted exosomes were resuspended in a small amount of PBS. The CM, exosomes, and exosome-free CM were stored after being dispensed in amounts of $1 \times 10^{6}$ cells.

Animal care and unilateral ureteral obstruction (UUO) mouse models. PDGFR-EGFP (enhanced green fluorescent protein) mice were obtained from Cyagen Biosciences; C57BL/6 mice were obtained from the Dalian Medical University Laboratory Animal Center and were housed at a constant room temperature with a 12-h light/dark cycle. Standard rodent chow and water were provided ad libitum. The animal experiments were conducted in accordance with the regulations established by the Institutional Committee for the Care and Use of Laboratory Animals and were approved by the Dalian Medical University Laboratory Animal Center; all efforts were made to minimize suffering. All surgical procedures were conducted by a single surgeon under aseptic conditions in the Laboratory Animal Unit. The mice were anesthetized with an intraperitoneal injection of freshly prepared $10 \%$ chloral hydrate. A midline incision was made in the abdominal wall, and the left ureter was isolated and ligated using a 4.0 silk 
suture at two points along its length. The wound was closed with a silk suture, and the mice were returned to their cages. The mice were humanely sacrificed on days 1,3 , and $7(n=3)$, and the kidneys were harvested for analysis.

\section{Mouse tail vein injection of MSCs, MSC-derived exosomes, CM, and exosome-free CM. After the UUO} mouse model had been constructed, the mice were divided into UUO, MSC, exosome, CM, and exosomefree $\mathrm{CM}$ groups. The mice from each group were fixed on the fixture. The tail was immersed in water at $40^{\circ} \mathrm{C}$ for $40 \mathrm{~s}$, and the tail hair was scraped with a blade to expose the tail vein. Using a 1-mL syringe, the reagent was slowly injected into the tail at about $30^{\circ} \mathrm{C}$ for about $1 \mathrm{~mm}$. After the injection, the injection site was pressed until the bleeding had stopped.

Renal histopathological staining. The mouse kidneys were fixed for $24 \mathrm{~h}$ in $10 \%$ formalin, removed, and dehydrated in alcohol series: $70 \%, 80 \%$, and $95 \%$ alcohol solution for $30 \mathrm{~min}$ each; $100 \%$ alcohol solution for $30 \mathrm{~min}$ twice, and then cleared for $20 \mathrm{~min}$ in chloroform. The kidney tissue was then placed in a paraffin solution for $25 \mathrm{~min}$ for paraffin embedding. Using a microtome, the kidney tissue was cut into 1$\mu \mathrm{m}$ thick paraffin sections on glass slides, and placed in a $60^{\circ} \mathrm{C}$ incubator for $5 \mathrm{~h}$. Then, the specimens were dewaxed in xylene I and II for 20 min each, followed by immersion in absolute ethanol I and II for 10 min each. Subsequently, the specimens were washed in $95 \%, 90 \%, 80 \%$, and $70 \%$ alcohol for 5 min each. Masson pathological staining was performed as follows: the nuclei were stained with Weigert's iron hematoxylin for $5 \mathrm{~min}$; differentiated for a few seconds using 1\% hydrochloric acid alcohol, and rinsed in tap water for 2 min, returning to blue. Then, the specimens were stained using Ponceau-acid fuchsin for 6-14 min, and rinsed with distilled water before 4-min phosphomolybdic acid treatment, 6-min aniline blue counterstaining, and 1-min 1\% glacial acetic acid treatment. Finally, the specimens were placed in 95\% alcohol I and II, absolute ethanol I and II, and then xylene I and II for 5 min each, dehydrated and cleared, and sealed using neutral gum. For periodic acid-Schiff (PAS) staining, the specimens were treated using $1 \%$ periodate oxide for 5-10 min, stained with Schiff's solution for 10-30 min, and rinsed in running water. This was followed by hematoxylin staining for 2-5 min, treatment with $1 \%$ hydrochloric acid alcohol, washing with tap water, treatment with $1 \%$ ammonia back to blue, and washing with tap water. Finally, the specimens were placed in $95 \%$ alcohol I and II, absolute ethanol I and II, and xylene I and II each for 5 min, dehydrated and cleared, and sealed in neutral gum.

The extent of tubulointerstitial damage (including the number of inflammatory cells, renal tubule expansion/atrophy, and proliferation of renal interstitial fibrous tissue) was evaluated in each group of stained sections. There were $0-3$ levels of damage: 0 indicated normal ( 0 points); 1 indicated $<25 \%$ fibrosis ( 1 point); 2 indicated $25-50 \%$ fibrosis ( 2 points); and 3 indicated $>50 \%$ fibrosis ( 3 points). Three fields per slice were randomly selected under $\times 200$ magnification, and the results were expressed as the mean \pm standard deviation. All pathological sections were evaluated independently by three investigators in a double-blinded manner. 
Pericyte isolation and the establishment of a pericyte activation model. Here, we used a model we have described previously ${ }^{9}$, with some modifications. The kidney was diced and incubated with liberase $(0.5$ $\mathrm{mg} / \mathrm{mL}$, Roche, Mannheim, Germany) and DNase (100 U/mL, Roche) in Hank's buffered salt solution for $45 \mathrm{~min}$ at $37^{\circ} \mathrm{C}$. After centrifugation, the cells were resuspended in Hank's buffered salt solution, and filtered $(40 \mu \mathrm{m})$. Then, impurities were removed using $42 \%$ Percoll solution, and the cell layer was collected. Pericytes were purified by isolating PDGFR- $\beta+$ cells using magnetic-activated cell sorting (Miltenyi Biotec, Gladbach, Germany), and cultured in pericyte medium supplemented with $2 \%$ fetal bovine serum, $1 \%$ pericyte growth supplement, and 1\% penicillin/streptomycin (ScienCell, Carlsbad, CA, USA) at $37^{\circ} \mathrm{C}$ in a $5 \% \mathrm{CO}_{2}$ atmosphere with $90 \%$ humidity. The medium was changed every 3 days. Passage 1 or 2 cells were used for the experiments. Pericyte activation was induced using $5 \mathrm{ng} / \mathrm{mL}$ recombinant TGF- $\beta 1$.

Immunofluorescence (kidney tissue and pericytes). Freshly harvested mouse kidney tissues were fixed in $4 \%$ paraformaldehyde (PFA) for $24 \mathrm{~h}$. The tissues were successively dehydrated in $30 \%, 20 \%$, and $10 \%$ sucrose solutions for $1 \mathrm{~h}$ each, and embedded in optimal cutting temperature compound (Tissue-Tek, Sakura Finetek, Tokyo, Japan). Next, 4- $\mu$ m cryosections were collected on Superfrost Plus glass slides, rinsed with PBS, and permeabilized with $1 \%$ Triton X-100 for $5 \mathrm{~min}$. Then, the sections were blocked with blocking buffer (Vector Laboratories, Burlingame, CA, USA) for $1 \mathrm{~h}$. The sections were incubated with primary antibodies against PDGFRß (ab32570, Abcam, Cambridge, MA, USA), CD31 (ab24590, Abcam), aSMA (ab205718, Abcam), CD81 (D502Q, Cellsignaling technology,USA), EGFR (ab52894, Abcam), WNT5a (ab235996, Abcam), NOTCH3 (ab23426, Abcam), PKH67 (UR52303, Umibio,Shanghai,CHINA), and LCA (Lens culinaris lectin, b-1045, Vector Laboratories) for $1 \mathrm{~h}$ at room temperature, and then incubated overnight at $4^{\circ} \mathrm{C}$. Then, the sections were incubated with secondary antibodies for $1 \mathrm{~h}$ at room temperature. Subsequently, the sections were mounted with Fluorescent Mounting Media with DAPI (Abcam). Each section was observed under a confocal laser scanning microscope (Leica SP8, Leica, Wetzlar, Germany) at $\times 400$ magnification, if necessary. The primary antibodies were omitted for the negative controls.

The pericytes were fixed in freshly prepared 4\% PFA for 10 min at room temperature, washed three times with PBS, and each cover slip was incubated in $1 \%$ bovine serum albumin (BSA). Then, the cells were incubated with the same primary antibodies as above for $1 \mathrm{~h}$ at room temperature. After washing in PBS, the cells were incubated with secondary antibodies conjugated with fluorescein isothiocyanate (FITC) or Cy3 for $1 \mathrm{~h}$ at room temperature in a darkened humidified chamber. Finally, the preparations were washed with PBS and mounted in Fluorescent Mounting Medium with DAPI (Abcam). Images were acquired using a confocal laser scanning microscope (Leica SP8) at $\times 400$ magnification. The primary antibodies were omitted for the negative controls. 
Western blotting. Cells were collected and lysed in radioimmunoprecipitation assay (RIPA) buffer [1\% Triton X-100, $150 \mathrm{mmol} / \mathrm{L} \mathrm{NaCl}, 1 \mathrm{mmol} / \mathrm{L}$ EGTA, $50 \mathrm{mmol} / \mathrm{L}$ Tris-HCl, $0.1 \%$ sodium dodecyl sulfate (SDS), $1 \%$ sodium deoxycholate, phenylmethylsulfonyl fluoride (PMSF)] on ice for $20 \mathrm{~min}$. Protein concentrations were determined with a bicinchoninic acid protein assay kit (Thermo, Rockford, IL, USA). Proteins (equal amounts, $10 \mu \mathrm{g} / \mathrm{lane}$ ) were separated on 6-10\% SDS-PAGE (polyacrylamide gel electrophoresis) and electrotransferred to polyvinylidene difluoride (PVDF) membranes (Bio-Rad, Hercules, CA, USA) at $400 \mathrm{~mA}$ for $2 \mathrm{~h}$. The membranes were then incubated in blocking buffer (PBS) with $0.05 \%$ Tween 20 and $0.1 \% \mathrm{BSA}$ for $1 \mathrm{~h}$ at room temperature, and then incubated overnight at $4^{\circ} \mathrm{C}$ with primary antibodies against a-SMA (ab205718, Abcam), FUT8 (ab19874, Abcam), TGFßR (ab31013, Abcam), PDGFRß (ab32570, Abcam), SMAD2/3 (ab202445, Abcam), ERK1/2 (ab184699, Abcam), phosphorylated (P)-SMAD2/3 (ab272332, Abcam), P-ERK1/2 (ab214362, Abcam), SMAD6/7 (ap52036, Abcepta,Beijing,CHINA), BMP7 (ab129156, Abcam), NOTCH3 (ab23426, Abcam), WNT5a (ab235996, Abcam), PCP (ab205394, Abcam), BMP7 (ab129156, Abcam), EGFR (ab52894, Abcam), ERBB3 (D22C5, Cellsignaling), P-ERBB3 (d1b5, Cellsignaling), NOTCH3 (ab23426, Abcam), CD63 (ab217345, Abcam), CD81 (ab109201, Abcam), and JAGGED (ab7771, Abcam). The membranes were incubated with secondary antibodies for $1 \mathrm{~h}$ at room temperature. The blots were washed three times in $1 \times$ PBS-Tween 20solution, and incubated for 1 min with electrochemiluminescence (ECL) reagents (Amersham, Pittsburgh, PA, USA). Proteins were visualized with Super RX-N film (Fujifilm Corporation, Tokyo, Japan). Anti-GAPDH antibody was used as the loading control to normalize the protein levels detected.

Immunoprecipitation. Cell lysates were centrifuged (12,000 rpm for $20 \mathrm{~min}$ at $\left.4^{\circ} \mathrm{C}\right)$, and the supernatant was collected and precleared using Protein G PLUS-Agarose (Santa Cruz Biotechnology, Santa Cruz, CA, USA). The cell lysates $(500 \mu \mathrm{g})$ were then incubated with $2 \mu \mathrm{g}$ antibody against TGF $\beta R$, PDGFR , EGFR, WNT5A, NOTCH3, and BMP7 for $2 \mathrm{~h}$ at $4^{\circ} \mathrm{C}$ on a rocker platform (30 rocks $/ \mathrm{min}$ ). Protein-antibody complexes were collected with $20 \mu \mathrm{L}$ Protein G PLUS-Agarose on a rocker platform (30 rocks/min) overnight at $4^{\circ} \mathrm{C}$. The immunoprecipitates were washed three times with lysis buffer. Equal amounts (10 $\mu \mathrm{g} /$ lane) of proteins underwent $12 \%$ SDS-PAGE for lectin blotting (described below). The primary antibodies were omitted for the negative controls.

Lectin blotting. Immunoprecipitated EGFR, WNT5A, NOTCH3, BMP7, TGF $\beta$ R, and PDGFR 3 were separated using $12 \%$ SDS-PAGE and electrotransferred to PVDF membranes. The membranes were blocked with $5 \%$ BSA (w/v) in Tris-buffered saline containing $0.05 \%$ Tween 20 (TBST) overnight at $4^{\circ} \mathrm{C}$, and then incubated for $1 \mathrm{~h}$ at $23^{\circ} \mathrm{C}$ with TBST containing LCA-Biotin (b-1045, Vector Laboratories), which preferentially recognizes Fuc-1,6 GlcNAc. The blots were washed three times with 1× PBS-Tween solution and incubated for $1 \mathrm{~min}$ with ECL reagents (Amersham). The lectin-reactive proteins were visualized using Super RX-N film (Fujifilm Corporation). 
Enzyme activity detection. FUT8 activity was measured using a previously described method ${ }^{50}$. Cell lysates $(5 \mu \mathrm{g})$ as the enzyme source were added to assay buffer $(200 \mathrm{mM} 2-(\mathrm{N}-$ Morpholino)ethanesulfonic acid hydrate $\triangle \mathrm{MES} \otimes, 1 \%$ Triton X-100) supplemented with donor (500 $\mu \mathrm{M}$ GDPL-fucose) and substrate [50 $\mu \mathrm{M}$ GnGn-Asn-4-(2-pyridylamine) butylamine (PABA)]. The mixture was incubated at $37^{\circ} \mathrm{C}$ for $4 \mathrm{~h}$, and the reaction was stopped by heating at $100^{\circ} \mathrm{C}$ for $5 \mathrm{~min}$. The reaction solution was then centrifuged at $12,000 \times g$ for $10 \mathrm{~min}$, and $10 \mu \mathrm{L}$ reaction products underwent highperformance liquid chromatography (HPLC) with a fluorescence detector (Waters Corporation, Milford, MA, USA) at excitation and emission wavelengths of $320 \mathrm{~nm}$ and $400 \mathrm{~nm}$, respectively.

DNA and RNA transfection. GP-transfect-Mate(GenePharma, Suzhou, China) was placed at room temperature before being mixed gently. Serum-free medium $(50 \mu \mathrm{L})$ was added into a 1.5-mL sterile centrifuge tube, and the transfection reagents was added, gently pipette-mixed, and stood at room temperature for $5 \mathrm{~min}$. At the same time, $50 \mu \mathrm{L}$ serum-free medium was added into another $1.5-\mathrm{mL}$ sterile centrifuge tube, and the DNA/RNA oligo was added, and pipette-mixed gently. The GP-transfect-Mate medium mixture was added to the DNA/RNA oligo medium mixture at room temperature for $5 \mathrm{~min}$. The mixture was lightly mixed with a transfer gun and transfected immediately after $15 \mathrm{~min}$ at room temperature. DNA/RNA expression was detected after 24-72-h transfection of $100 \mu \mathrm{L}$ compound.

Inhibition of CD81 and EGFR expression. Adenoviruses carrying CD81 short hairpin RNA (shRNA) and EGFR shRNA were constructed commercially (GenePharma, Suzhou, China). The shRNA-carrying adenoviruses were administered to the mice via tail vein injection of $1 \times 10^{6}$ plaque-forming units (PFU) before anesthesia for UUO surgery. The shRNAs were designed for knockdown in pericytes in vitro. To confirm the knockdown, we extracted RNA from the cells and assessed CD81 and EGFR expression using reverse transcriptase-PCR (RT-PCR) according to the manufacturer's protocol.

RT-PCR. RNA was extracted from the cells, and the RNA concentration was measured. Then, the genomic DNA was removed, and the complementary DNA (CDNA) was reverse-transcribed using a Verso cDNA Synthesis Kit (Thermo Fisher Scientific) using EvaGreen qPCR Supermix (Solis BioDyne,Shanghai,CHINA), followed by PCR.

Flow cytometry. Cells were fixed with $0.05 \%$ glutaraldehyde (Sigma,USA) for $10 \mathrm{~min}$ at room temperature, and then permeabilized with $0.1 \%$ Triton X-100 (Life Technologies,USA) for $5 \mathrm{~min}$ at room temperature. The cells were transfected with fluorescent protein/microRNA (miRNA) via a transfection 
kit(GenePharma, Suzhou, China), then washed with PBS three times to prepare single-cell suspensions. The fluorescence signals were collected from the suspensions, and the results were analyzed statistically.

Statistical analysis. Data are presented as the means \pm standard deviations. Multiple comparisons of parametric data were performed using one-way analysis of variance (ANOVA). Nonparametric data were compared with the Mann-Whitney U-test to identify differences between groups. Pearson's correlation analysis was calculated to determine the associations. $P<0.05$ was considered to indicate statistical significance. All statistical analyses were performed using SPSS version 21.0 (IBM, Inc., Armonk, NY, USA).

\section{References}

1. Zhang L, et al. Prevalence of chronic kidney disease in China: a cross-sectional survey. The Lancet 379, 815-822 (2012).

2. LeBleu VS, et al. Origin and function of myofibroblasts in kidney fibrosis. Nature Medicine 19, 1047 (2013).

3. DiRocco DP, Kobayashi A, Taketo MM, McMahon AP, Humphreys BD. Wnt4/ $\beta$-Catenin Signaling in Medullary Kidney Myofibroblasts. Journal of the American Society of Nephrology 24, 1399-1412 (2013).

4. Kawakami T, Mimura I, Shoji K, Tanaka T, Nangaku M. Hypoxia and fibrosis in chronic kidney disease: crossing at pericytes. Kidney International Supplements 4, 107-112 (2014).

5. Duffield JS. Cellular and molecular mechanisms in kidney fibrosis. The Journal of Clinical Investigation 124, 2299-2306 (2014).

6. Wang N, et al. Novel Mechanism of the Pericyte-Myofibroblast Transition in Renal Interstitial Fibrosis: Core Fucosylation Regulation. Scientific reports 7, (2017). 
7. Shen N, et al. Inhibition of TGF- $\beta 1$-receptor posttranslational core fucosylation attenuates rat renal interstitial fibrosis. Kidney International 84, 64-77 (2013).

8. Wang $\mathrm{N}$, et al. Novel mechanism for mesenchymal stem cells in attenuating peritoneal adhesion: accumulating in the lung and secreting tumor necrosis factor a-stimulating gene-6. Stem Cell Res Ther 3, 51 (2012).

9. Chen Y-T, et al. Platelet-derived growth factor receptor signaling activates pericyte-myofibroblast transition in obstructive and post-ischemic kidney fibrosis. Kidney Internationa/ 80, 1170-1181 (2011).

10. Garcia I, Arenas-Alfonseca L, Moreno I, Gotor C, Romero LC. HCN Regulates Cellular Processes through Posttranslational Modification of Proteins by S-cyanylation. Plant physiology 179, 107-123 (2019).

11. Ihara $\mathrm{H}$, Ikeda $\mathrm{Y}$, Taniguchi $\mathrm{N}$. Reaction mechanism and substrate specificity for nucleotide sugar of mammalian a1,6-fucosyltransferase-a large-scale preparation and characterization of recombinant human FUT8. Glycobiology 16, 333-342 (2006).

12. Ihara $\mathrm{H}$, et al. Crystal structure of mammalian a1,6-fucosyltransferase, FUT8. Glycobiology 17, 455466 (2007).

13. Chuang HM, Shih TE, Lu KY, Tsai SF, Harn HJ, Ho LI. Mesenchymal Stem Cell Therapy of Pulmonary Fibrosis: Improvement with Target Combination. Cell Transplant, 963689718787501 (2018).

14. Bianchi F, Sala E, Donadei C, Capelli I, La Manna G. Potential advantages of acute kidney injury management by mesenchymal stem cells. World Journal of Stem Cells 6, 644-650 (2014). 
15. Lou G, Chen Z, Zheng M, Liu Y. Mesenchymal stem cell-derived exosomes as a new therapeutic strategy for liver diseases. Experimental \& Molecular Medicine 49, e346 (2017).

16. Mishra VK, et al. Identifying the Therapeutic Significance of Mesenchymal Stem Cells. Cells $\mathbf{9}, 1145$ (2020).

17. He J, et al. Bone marrow stem cells-derived microvesicles protect against renal injury in the mouse remnant kidney model. Nephrology 17, 493-500 (2012).

18. Wang $\mathrm{Y}$, et al. Exosomes from embryonic mesenchymal stem cells alleviate osteoarthritis through balancing synthesis and degradation of cartilage extracellular matrix. Stem Cell Res Ther $\mathbf{8}, 189$ (2017).

19. Nolan-Stevaux O, et al. Differential Contribution to Neuroendocrine Tumorigenesis of Parallel Egfr Signaling in Cancer Cells and Pericytes. Genes \& Cancer 1, 125-141 (2010).

20. Zou G-L, et al. Bone morphogenetic protein-7 represses hepatic stellate cell activation and liver fibrosis via regulation of TGF- $\beta /$ Smad signaling pathway. World Journal of Gastroenterology 25 , 4222-4234 (2019).

21. Liu H, Zhang W, Lilly B. Evaluation of $\langle\mathrm{b}><\mathrm{i}>$ Notch3 $</ \mathrm{i}></ \mathrm{b}>$ Deficiency in Diabetes-Induced Pericyte Loss in the Retina. Journal of Vascular Research 55, 308-318 (2018).

22. Yuan K, et al. Loss of Endothelium-Derived Wnt5a Is Associated With Reduced Pericyte Recruitment and Small Vessel Loss in Pulmonary Arterial Hypertension. Circulation 139, 1710-1724 (2019).

23. Ying W, et al. Proteomic analysis on structural proteins of Severe Acute Respiratory Syndrome coronavirus. Proteomics 4, 492-504 (2004). 
24. Valadi H, Ekström K, Bossios A, Sjöstrand M, Lee JJ, Lötvall JO. Exosome-mediated transfer of mRNAs and microRNAs is a novel mechanism of genetic exchange between cells. Nature Cell Biology 9, 654 (2007).

25. Qu Y, et al. Exosomes derived from miR-181-5p-modified adipose-derived mesenchymal stem cells prevent liver fibrosis via autophagy activation. J Cell Mol Med 21, 2491-2502 (2017).

26. Wang B, et al. Mesenchymal Stem Cells Deliver Exogenous MicroRNA-let7c via Exosomes to Attenuate Renal Fibrosis. Molecular therapy : the journal of the American Society of Gene Therapy 24, 1290-1301 (2016).

27. Ohno S, et al. Systemically injected exosomes targeted to EGFR deliver antitumor microRNA to breast cancer cells. Molecular therapy : the journal of the American Society of Gene Therapy 21, 185-191 (2013).

28. Diao J, et al. Hepatitis $\mathrm{C}$ virus induces epidermal growth factor receptor activation via CD81 binding for viral internalization and entry. Journal of virology 86, 10935-10949 (2012).

29. da Silva AF, Silva K, Reis LA, Teixeira VPC, Schor N. Bone Marrow-Derived Mesenchymal Stem Cells and Their Conditioned Medium Attenuate Fibrosis in an Irreversible Model of Unilateral Ureteral Obstruction. 24, 2657-2666 (2015).

30. Liu B, et al. Human umbilical cord mesenchymal stem cell conditioned medium attenuates renal fibrosis by reducing inflammation and epithelial-to-mesenchymal transition via the TLR4/NF-kappaB signaling pathway in vivo and in vitro. Stem Cell Res Ther 9, 7 (2018).

31. Sung P-H, et al. Apoptotic adipose-derived mesenchymal stem cell therapy protects against lung and kidney injury in sepsis syndrome caused by cecal ligation puncture in rats. Stem Cell Res Ther 4, 155155 (2013). 
32. Duffield JS, et al. Restoration of tubular epithelial cells during repair of the postischemic kidney occurs independently of bone marrow-derived stem cells. The Journal of Clinical Investigation 115, 1743-1755 (2005).

33. Li D, et al. Mesenchymal stem cells protect podocytes from apoptosis induced by high glucose via secretion of epithelial growth factor. Stem Cell Res Ther 4, 103-103 (2013).

34. Kinnaird T. Bone Marrow-Derived Cells for Enhancing Collateral Development: Mechanisms, Animal Data, and Initial Clinical Experiences. Circulation Research 95, 354-363 (2004).

35. Hu J, et al. Mesenchymal stem cells attenuate ischemic acute kidney injury by inducing regulatory $\mathrm{T}$ cells through splenocyte interactions. Kidney Internationa/ 84, 521-531 (2013).

36. Camussi G, Deregibus MC, Bruno S, Cantaluppi V, Biancone L. Exosomes/microvesicles as a mechanism of cell-to-cell communication. Kidney International 78, 838-848 (2010).

37. Marote A, Teixeira FG, Mendes-Pinheiro B, Salgado AJ. MSCs-Derived Exosomes: Cell-Secreted Nanovesicles with Regenerative Potential. Frontiers in Pharmacology 7, (2016).

38. Aghajani Nargesi A, O. Lerman L, Eirin A. Mesenchymal Stem Cell-derived Extracellular Vesicles for Renal Repair. Current Gene Therapy 17, 29-42 (2017).

39. Lu K, et al. Exosomes as potential alternatives to stem cell therapy for intervertebral disc degeneration: in-vitro study on exosomes in interaction of nucleus pulposus cells and bone marrow mesenchymal stem cells. Stem Cell Res Ther 8, 108 (2017).

40. Weng $Y$, et al. Effective isolation of exosomes with polyethylene glycol from cell culture supernatant for in-depth proteome profiling. The Analyst 141, 4640-4646 (2016). 
41. Bruno $S$, et al. Mesenchymal stem cell-derived microvesicles protect against acute tubular injury. $J$ Am Soc Nephrol 20, 1053-1067 (2009).

42. Grange $\mathrm{C}$, et al. Biodistribution of mesenchymal stem cell-derived extracellular vesicles in a model of acute kidney injury monitored by optical imaging. Int J Mol Med 33, 1055-1063 (2014).

43. Herrera MB, et al. Exogenous mesenchymal stem cells localize to the kidney by means of CD44 following acute tubular injury. Kidney Int 72, 430-441 (2007).

44. Caja $L$, et al. Snail regulates BMP and TGF $\beta$ pathways to control the differentiation status of gliomainitiating cells. Oncogene 37, 2515-2531 (2018).

45. Wang B, et al. Mesenchymal Stem Cells Deliver Exogenous MicroRNA-let7c via Exosomes to Attenuate Renal Fibrosis. Molecular Therapy 24, 1290-1301 (2016).

46. Baglio SR, et al. Human bone marrow- and adipose-mesenchymal stem cells secrete exosomes enriched in distinctive miRNA and tRNA species. Stem Cell Res Ther 6, 127 (2015).

47. Quan Y, et al. Exosome miR-371b-5p promotes proliferation of lung alveolar progenitor type Il cells by using PTEN to orchestrate the PI3K/Akt signaling. Stem Cell Res Ther 8, 138 (2017).

48. Morizane R, et al. miR-34c attenuates epithelial-mesenchymal transition and kidney fibrosis with ureteral obstruction. Scientific reports 4, 4578 (2014).

49. Sharma NR, Mateu G, Dreux M, Grakoui A, Cosset F-L, Melikyan GB. Hepatitis C Virus Is Primed by CD81 Protein for Low pH-dependent Fusion. Journal of Biological Chemistry 286, 30361-30376 (2011). 
50. Liang W, et al. Core Fucosylation of the T Cell Receptor Is Required for T Cell Activation. Frontiers in Immunology 9, 78 (2018).

\section{Declarations}

Acknowledgements This research was supported by grants from the following agencies: 1 . The NSFC, No. 81530021; 2. The Natural Science Foundation of China(NSFC), No. 81470996; 3. The NSFC, No. 81770694; 4. The Science and TechnologyTalent Training Plan of the First Affiliated Hospital of Dalian Medical University, No. 2015 YC002.

Author contributions X.H., W.W.,Q.T., X.D.,B.Q. Z.L. and L.L. performed the animal experiments, PCR, IHC, IF and Western blot experiments, and collected and analyzed the data. X.H., A.L.,L.Z.,Z.S.,W.Y., performed the cell-based experiments and collected and analyzed the data. H.L.,N.W.,X.H., N.S., N.Y. and designed the study, interpreted the results, wrote the paper,revised the paper and discussed analyses. All authors have contributed to read and approved the manuscript.

Competing interests The authors declare no competing interests.

\section{Figures}



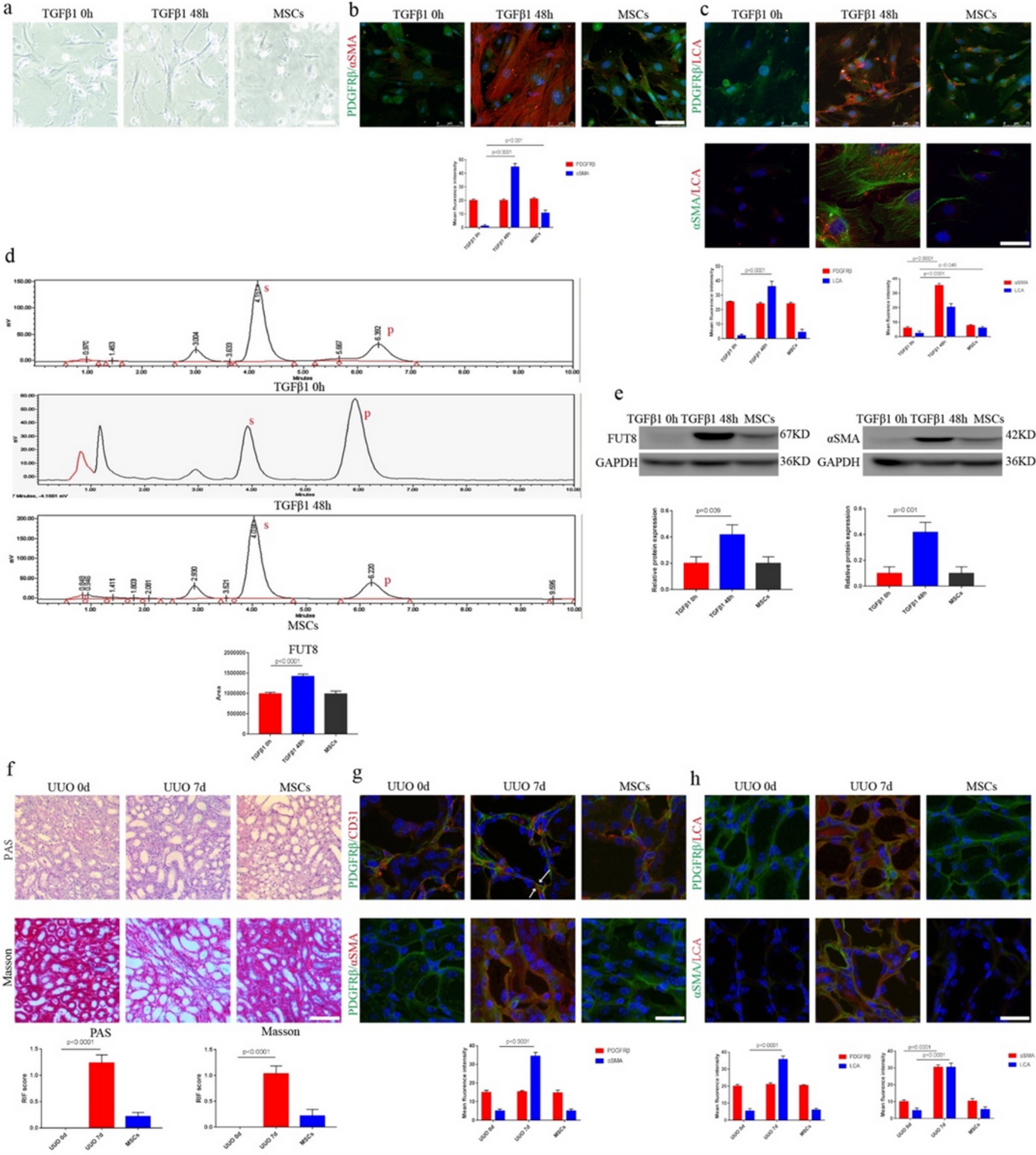

\section{Figure 1}

MSCs inhibited CF during pericyte activation. a Representative morphological alterations in pericytes. Scale bar, $200 \mu \mathrm{m}$. b Representative images of PDGFRß (green) and a-SMA (red) staining. The bottom panel shows the quantification. Scale bar, $75 \mu \mathrm{m}$. Data are the mean $\pm S D(n=3)$. c Representative images of PDGFRß (green) and LCA (red) staining, and a-SMA (green) and LCA (red) staining. The bottom panel shows the quantification. Scale bar, $75 \mu \mathrm{m}$. Data are the mean \pm SD $(n=3)$. d FUT8 activity tested 
by HPLC. Data are the mean $\pm S D(n=3)$; $S$ indicates the peptide substrate; $P$ is the fucosylation product. e a-SMA and FUT8 levels were assessed using western blotting. The bottom panel shows the quantification. Scale bar, $50 \mu \mathrm{m}$. Data are the mean \pm SD $(n=3)$. MSCs, 48-h TGF- $\beta 1-$ stimulated pericytes plus MSCs. f Representative images of Masson's trichrome and PAS staining of UUO mouse kidney sections. The bottom panel shows the quantification. Scale bar, $200 \mu \mathrm{m}$. Data are the mean $\pm S D$ $(n=3)$. g Representative images of PDGFR (green) and CD31 (red) staining, and PDGFRß (green) and aSMA (red) staining. The bottom panel shows the quantification. Scale bar, $75 \mu \mathrm{m}$. Data are the mean \pm SD $(n=3)$. h Representative images of PDGFR (green) and LCA (red) staining, and a-SMA (green) and LCA (red) staining. The bottom panel shows the quantification. Scale bar, $75 \mu \mathrm{m}$. Data are the mean $\pm S D(n=$ 3). MSCs, 7 days after MSC injection into UUO mouse tail vein. 
a
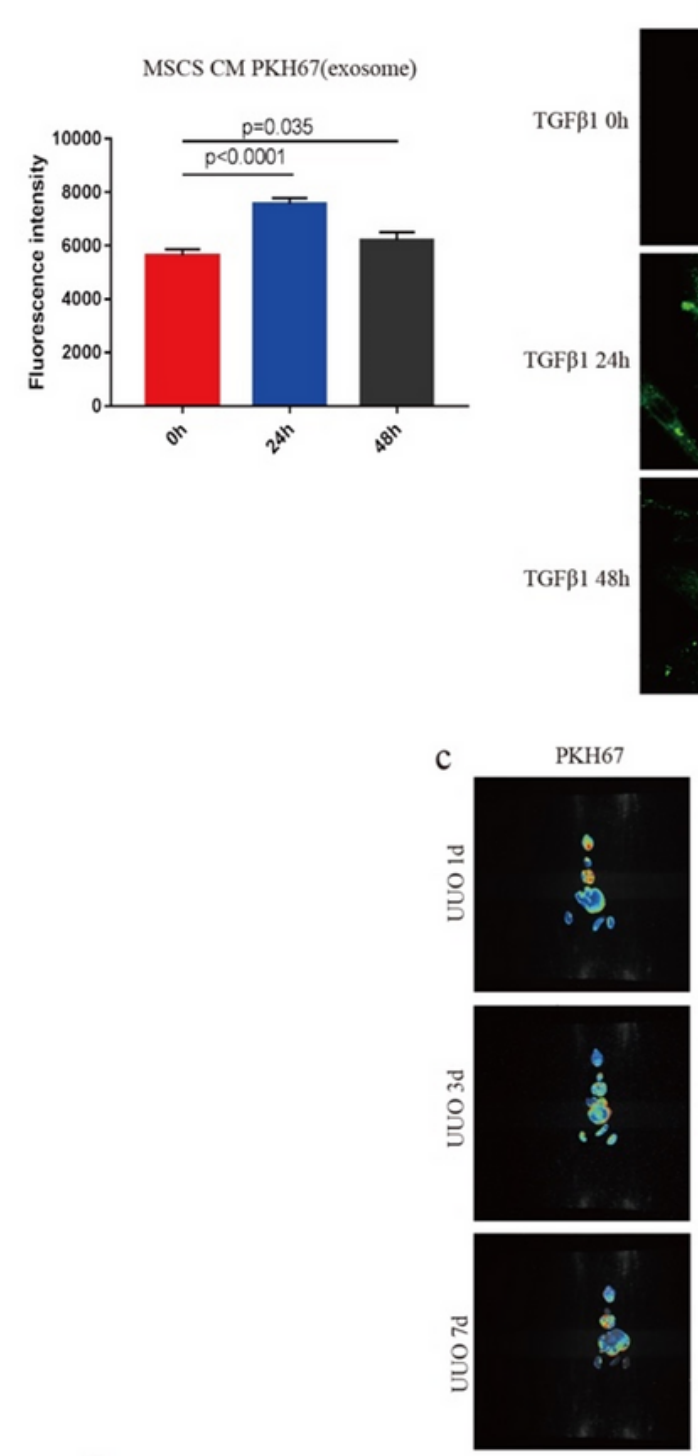

d

UUO 0d

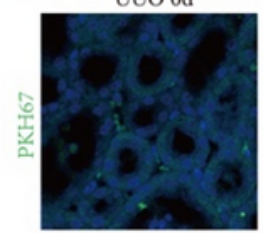

UUO 7d
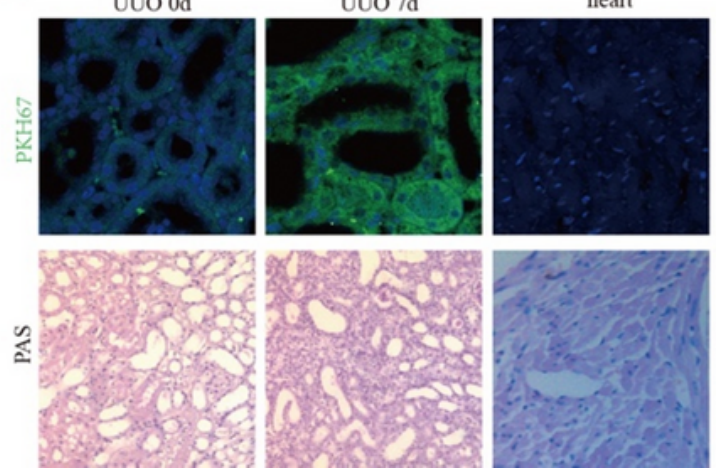

b
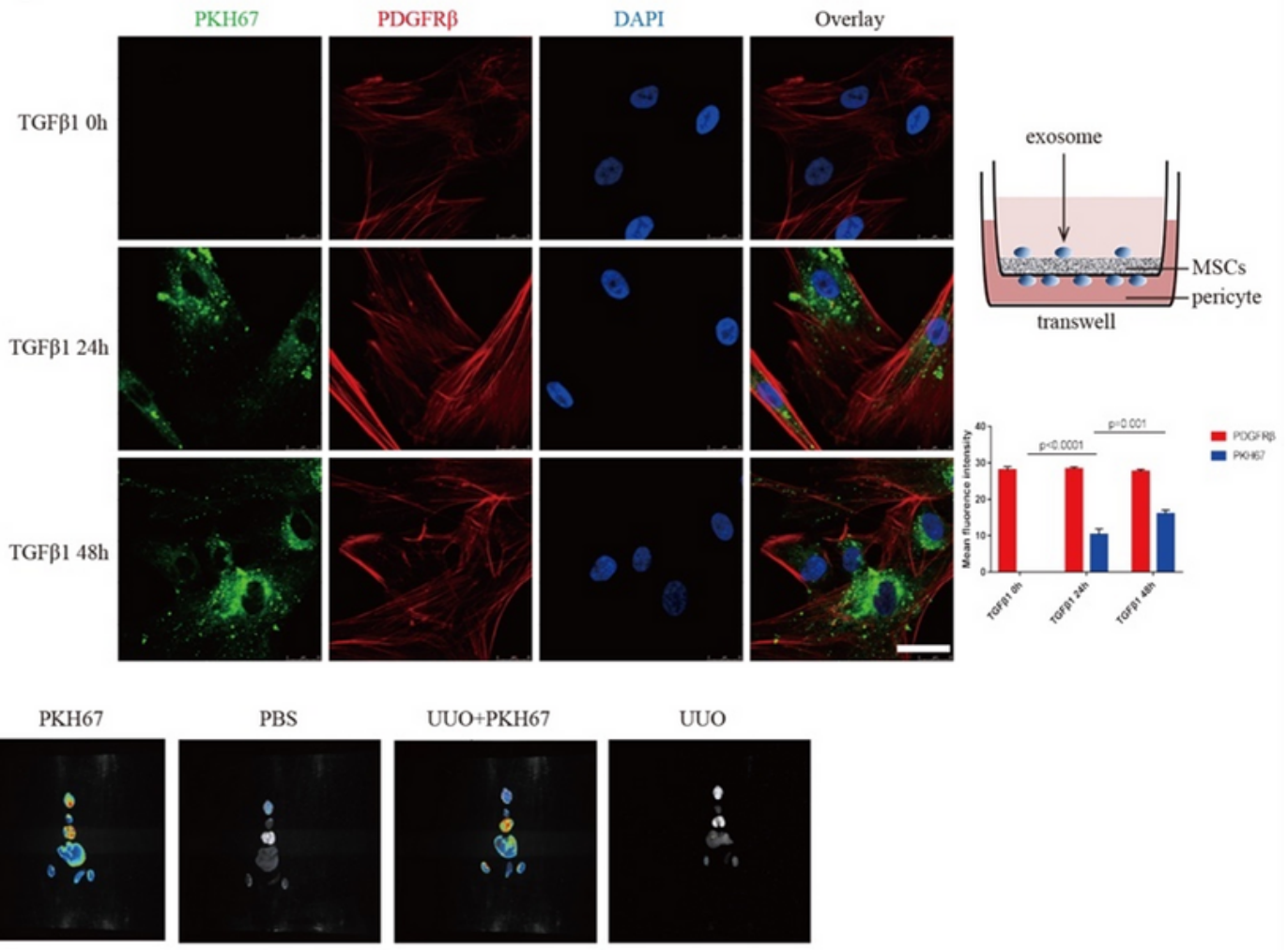

UUO+PKH67

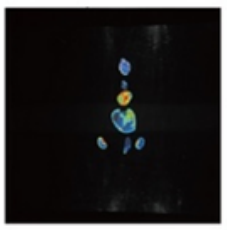

UUO
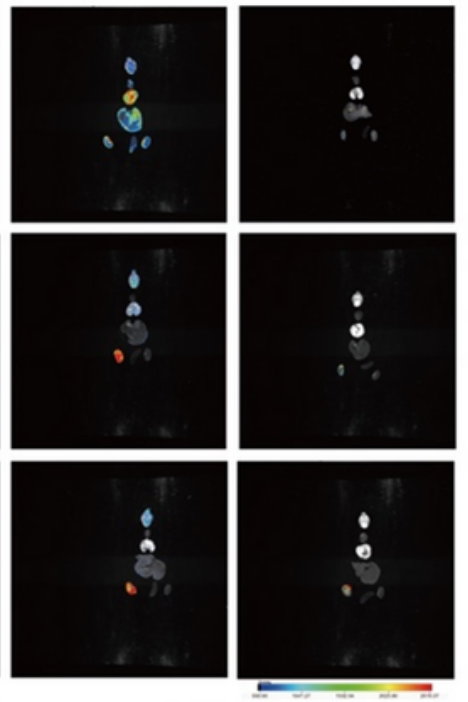

spleen
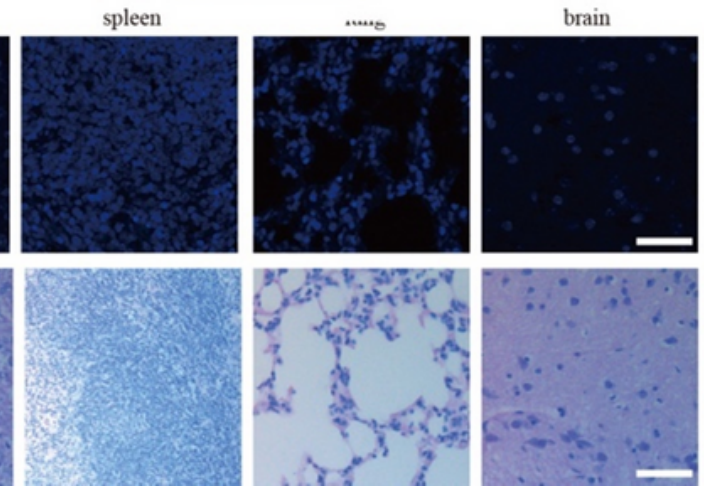

\section{Figure 2}

Exosomes accumulated mainly in the obstructed kidney. a The exosome content of MSC CM was detected by luciferase. Data are the mean \pm SD $(n=3)$. b Representative images of PKH67 (green) and PDGFR $\beta$ (red) staining in vitro. The right panel shows the quantification. Scale bar, $75 \mu \mathrm{m}$. Data are the mean $\pm S D(n=3)$. c In vivo UUO mouse imaging . $d$ Representative images of PKH67 (green) staining in 
vivo. Scale bar, $75 \mu \mathrm{m}$. Also shown are representative images of PAS-stained sections from the heart, spleen, liver, lung, and brain ( $\mathrm{n}=3)$. Scale bar, $200 \mu \mathrm{m}$.

a

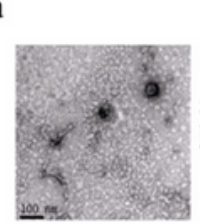

C
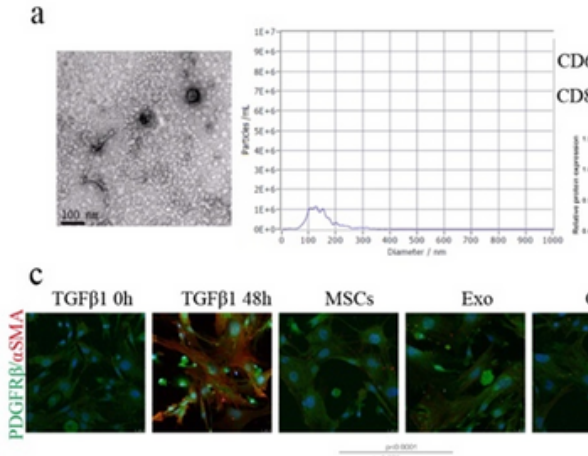

CM-Exo Exo

b
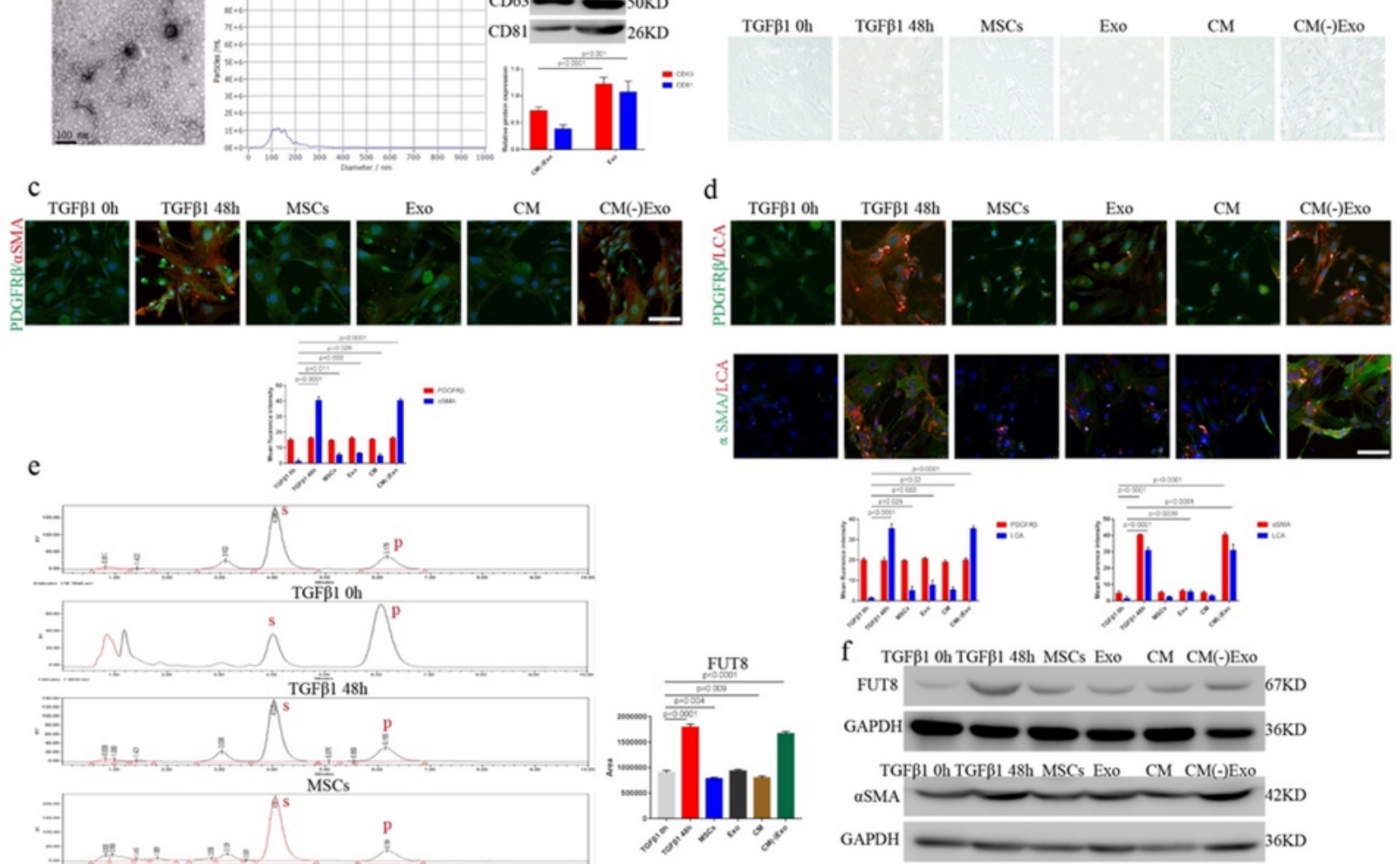

f TGFß1 0h TGFß1 48h MSCs Exo CM CM(-)Exo
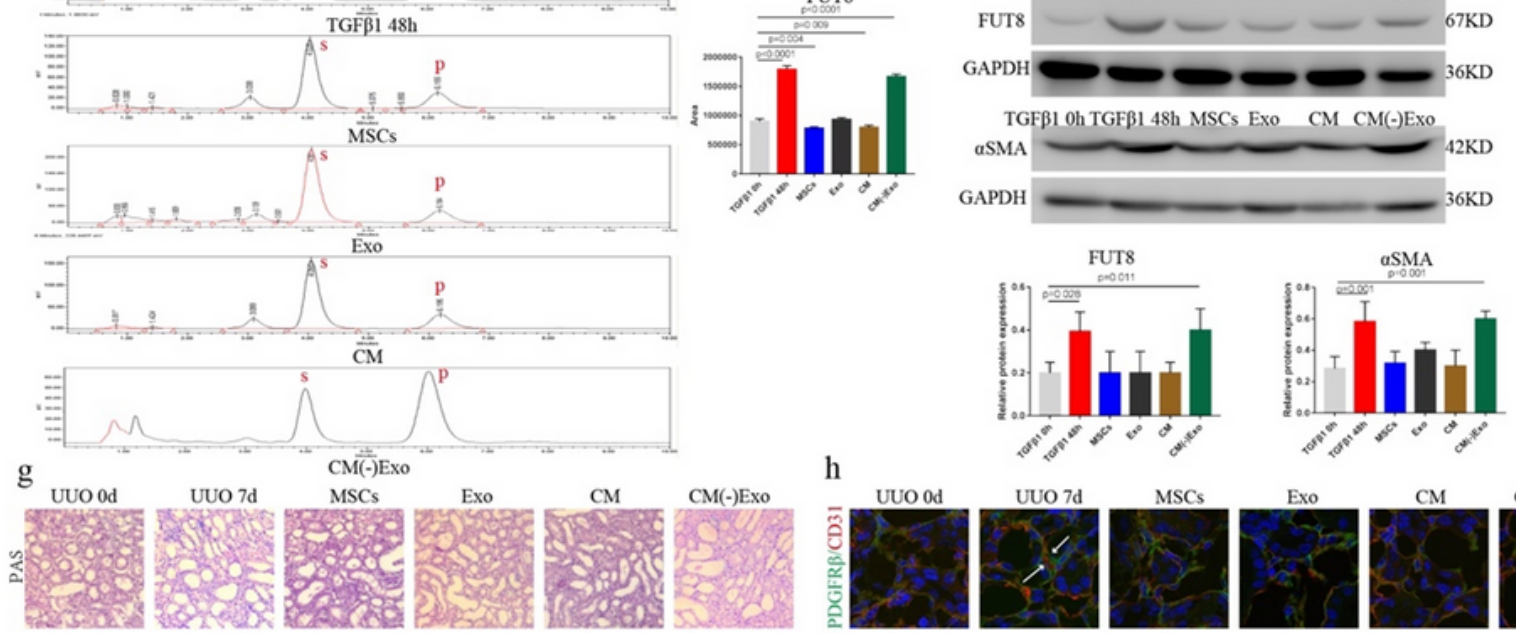

h
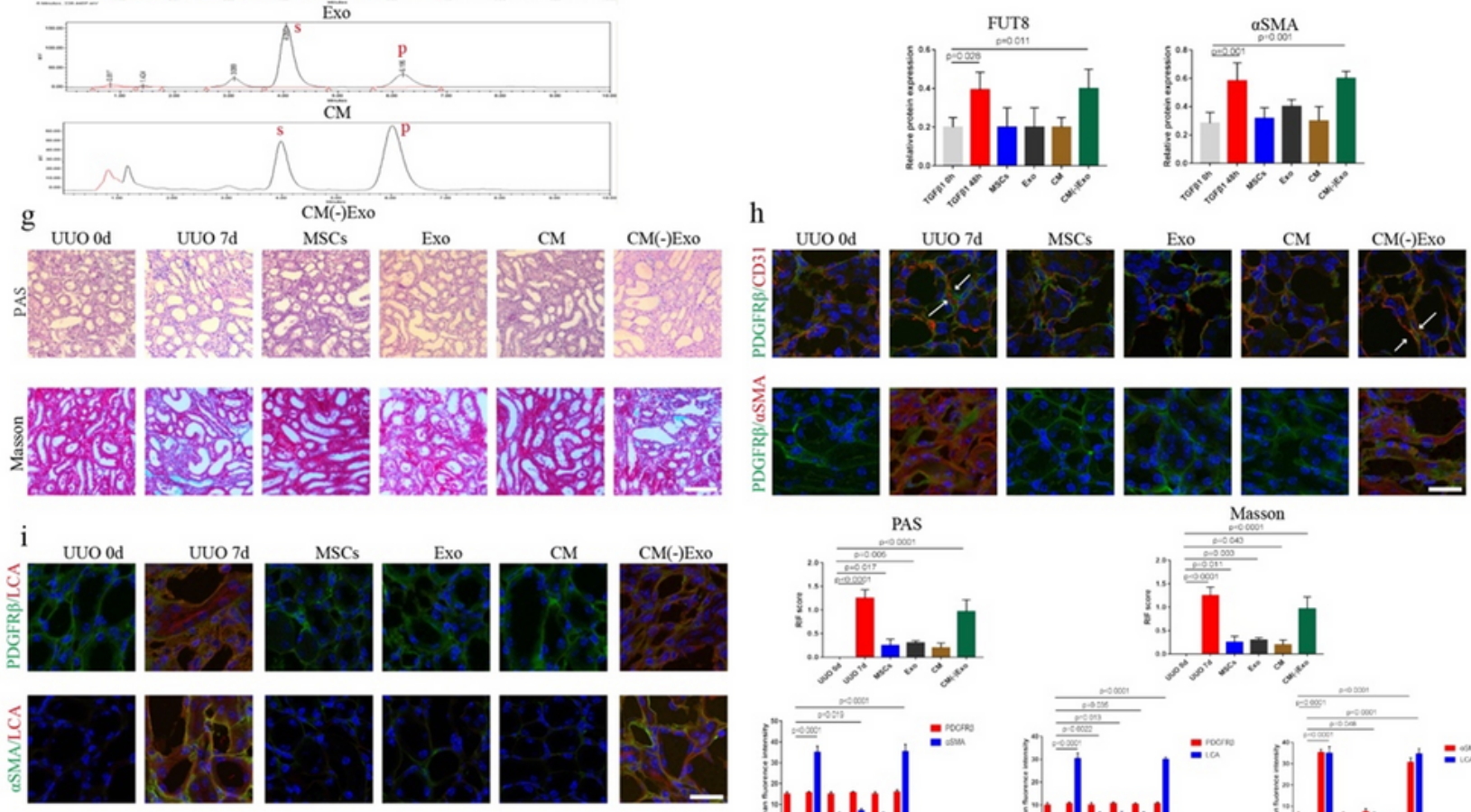

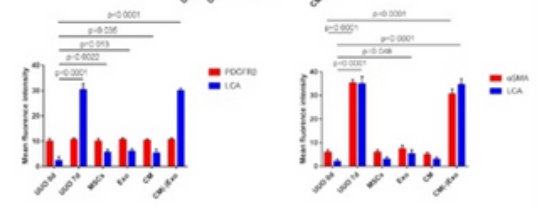

Figure 3

MSC-derived exosomes inhibited CF and ameliorated pericyte activation. a (Left) Representative electron micrograph of exosomes. Scale bar, $100 \mu \mathrm{m}$. (Middle) Nanoparticle tracking analysis. (Right) CD63 and CD81 levels were assessed using western blotting. The bottom panel shows the quantification. Scale bar, 
$50 \mu \mathrm{m}(\mathrm{n}=3)$. b Representative morphological alterations in pericytes. Scale bar, $200 \mu \mathrm{m}$. c Representative images of PDGFRß (green) and a-SMA (red) staining. The bottom panel shows the quantification. Scale bar, $75 \mu \mathrm{m}$. d Representative images of PDGFRß (green) and LCA (red) staining, and a-SMA (green) and LCA (red) staining. The bottom panel shows the quantification. Scale bar, $75 \mu \mathrm{m}$. Data are the mean $\pm S D(n=3)$. e FUT8 activity tested by HPLC. Data are the mean $\pm S D(n=3) ; S$ indicates the peptide substrate; $P$ is the fucosylation product. $\mathrm{f}$ a-SMA and FUT8 levels were assessed using western blotting. The bottom panel shows the quantification. Scale bar, $50 \mu \mathrm{m}$. Data are the mean $\pm S D(n=3)$. MSCs, 48-h TGF- $\beta 1$-stimulated pericytes plus MSCs; Exo, 48-h TGF- $\beta 1$-stimulated pericytes plus exosomes; CM, 48-h TGF- $\beta 1$-stimulated pericytes plus CM; CM(-)Exo, 48-h TGF- $\beta 1$-stimulated pericytes plus CM(-)Exo. g Representative images of Masson's trichrome and PAS staining of UUO mouse kidney sections. The bottom panel shows the quantification. Scale bar, $200 \mu \mathrm{m}$. Data are the mean $\pm S D(n=3)$. h Representative images of PDGFR 3 (green) and CD31 (red) staining, and PDGFR 3 (green) and a-SMA (red) staining. The bottom panel shows the quantification. Scale bar, $75 \mu \mathrm{m}$. Data are the mean $\pm S D(n=$ 3). i Representative images of PDGFR $\beta$ (green) and LCA (red) staining, and a-SMA (green) and LCA (red) staining. The bottom right panel shows the quantification. Scale bar, $75 \mu \mathrm{m}$. Data are the mean $\pm S D(n=$ 3). MSCs, MSC-injected UUO mouse tail vein after 7 days; Exo, Exo-injected UUO mouse tail vein after 7 days; CM, CM-injected UUO mouse tail vein after 7 days; CM(-)Exo, CM(-)Exo-injected UUO mouse tail vein after 7 days. 

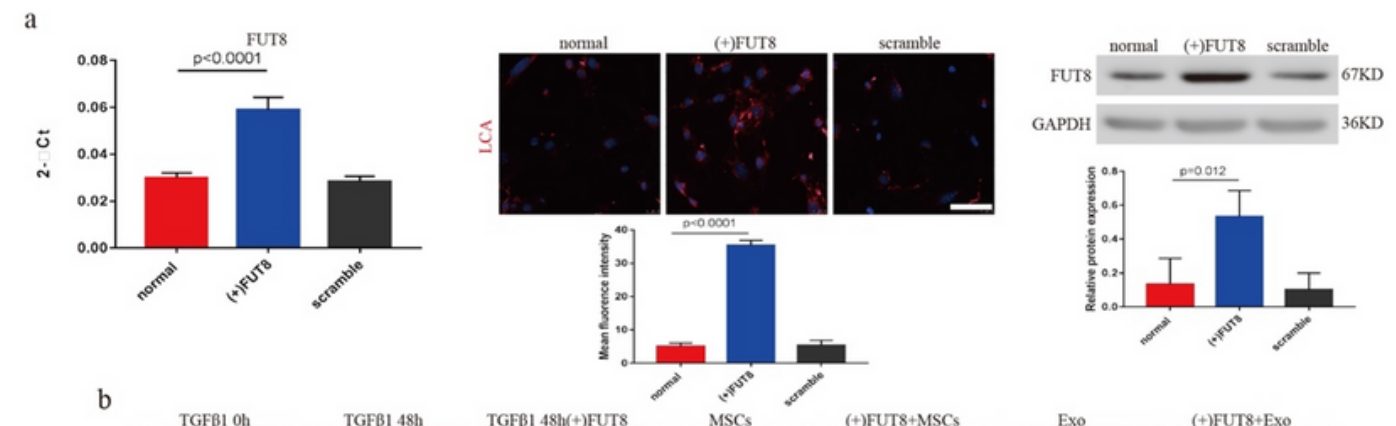

b
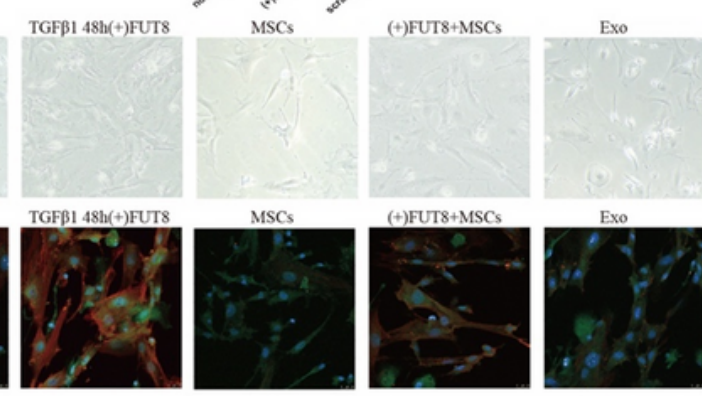

(+)FUT8+Exo
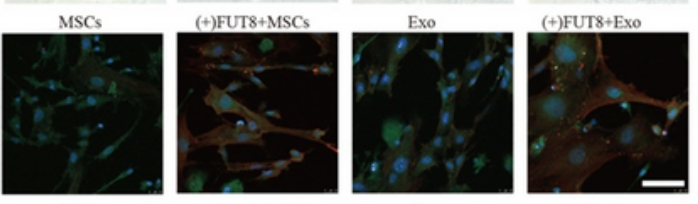

d

TGFB1 48h
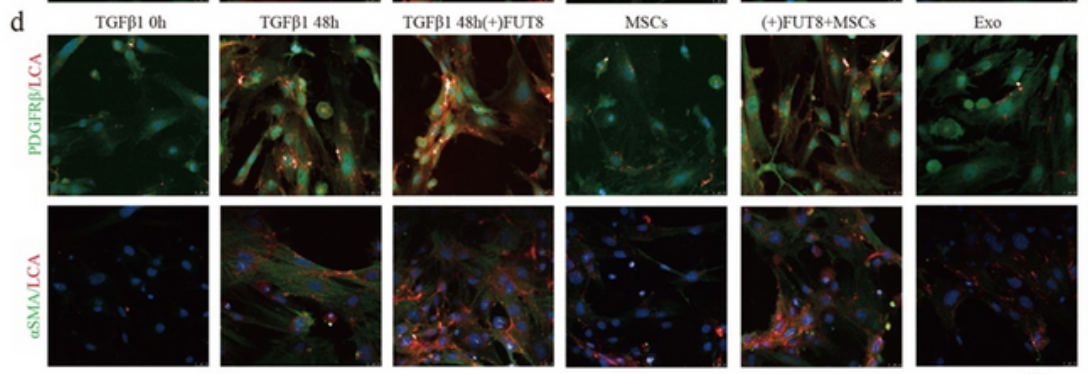

(+)FUT8+Exo
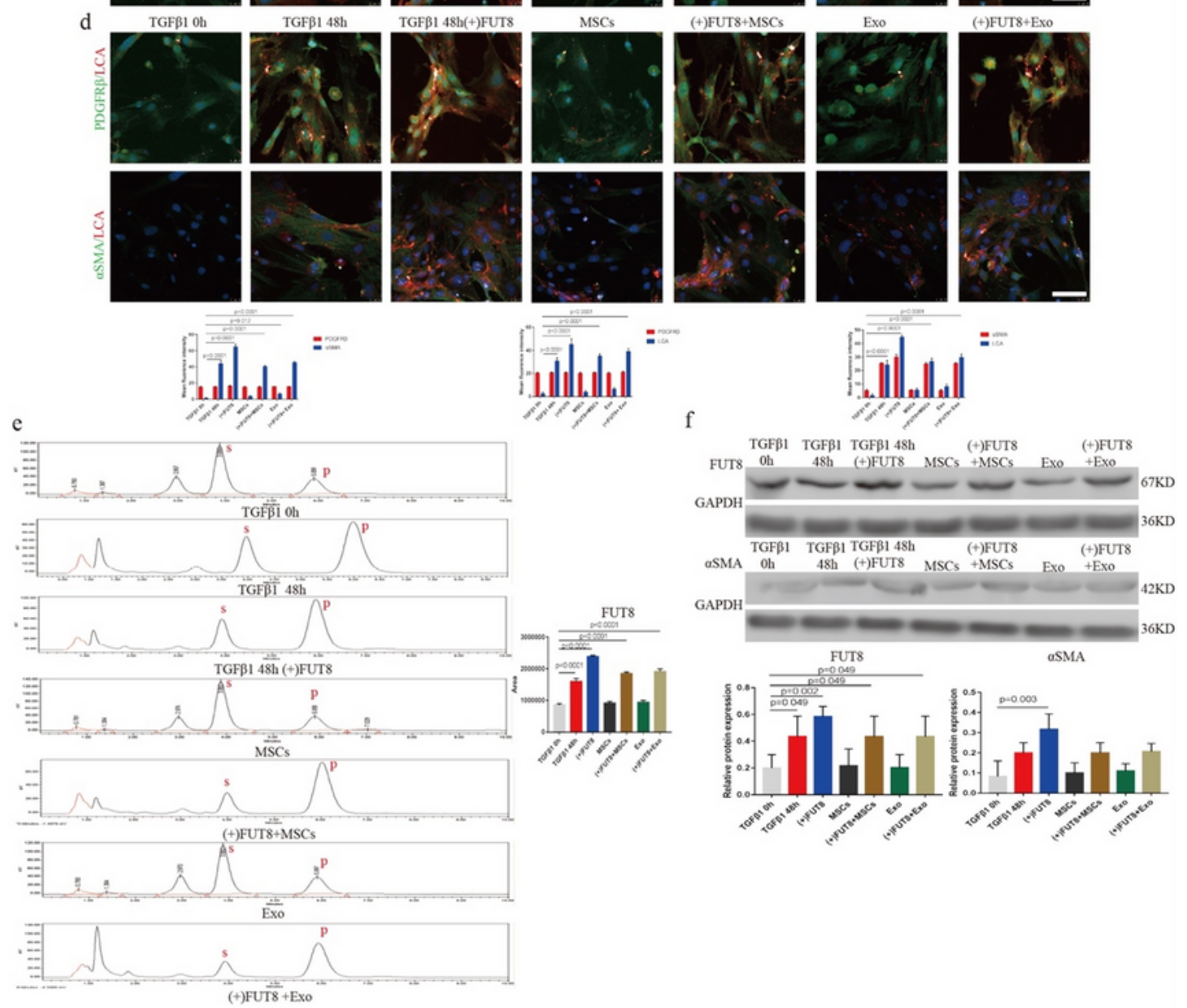

\section{Figure 4}

MSC-derived exosomes ameliorated pericyte activation and RIF by inhibiting CF. a (Left) RT-PCR, (middle) representative images of FUT8 (red) staining, (right) FUT8 levels assessed by western blotting. The bottom panel shows the quantification. Data are the mean $\pm S D(n=3)$. b Representative morphological alterations in pericytes. Scale bar, $200 \mu \mathrm{m}$. c Representative images of PDGFR (green) and a-SMA (red) staining. The bottom panel shows the quantification. Scale bar, $75 \mu \mathrm{m}$. Data are the mean $\pm S D(n=3)$. $d$ 
Representative images of PDGFR $\beta$ (green) and LCA (red) staining, and a-SMA (green) and LCA (red) staining. The panel shows the quantification. Scale bar, $75 \mu \mathrm{m}$. Data are the mean $\pm S D(n=3)$. e FUT8 activity tested by HPLC. Data are the mean $\pm S D(n=3)$; $S$ indicates the peptide substrate; $P$ is the fucosylation product. $\mathrm{f}$ a-SMA and FUT8 levels were assessed by western blotting. The bottom panel shows the quantification. Scale bar, $50 \mu \mathrm{m}$. Data are the mean $\pm \operatorname{SD}(n=3)$.

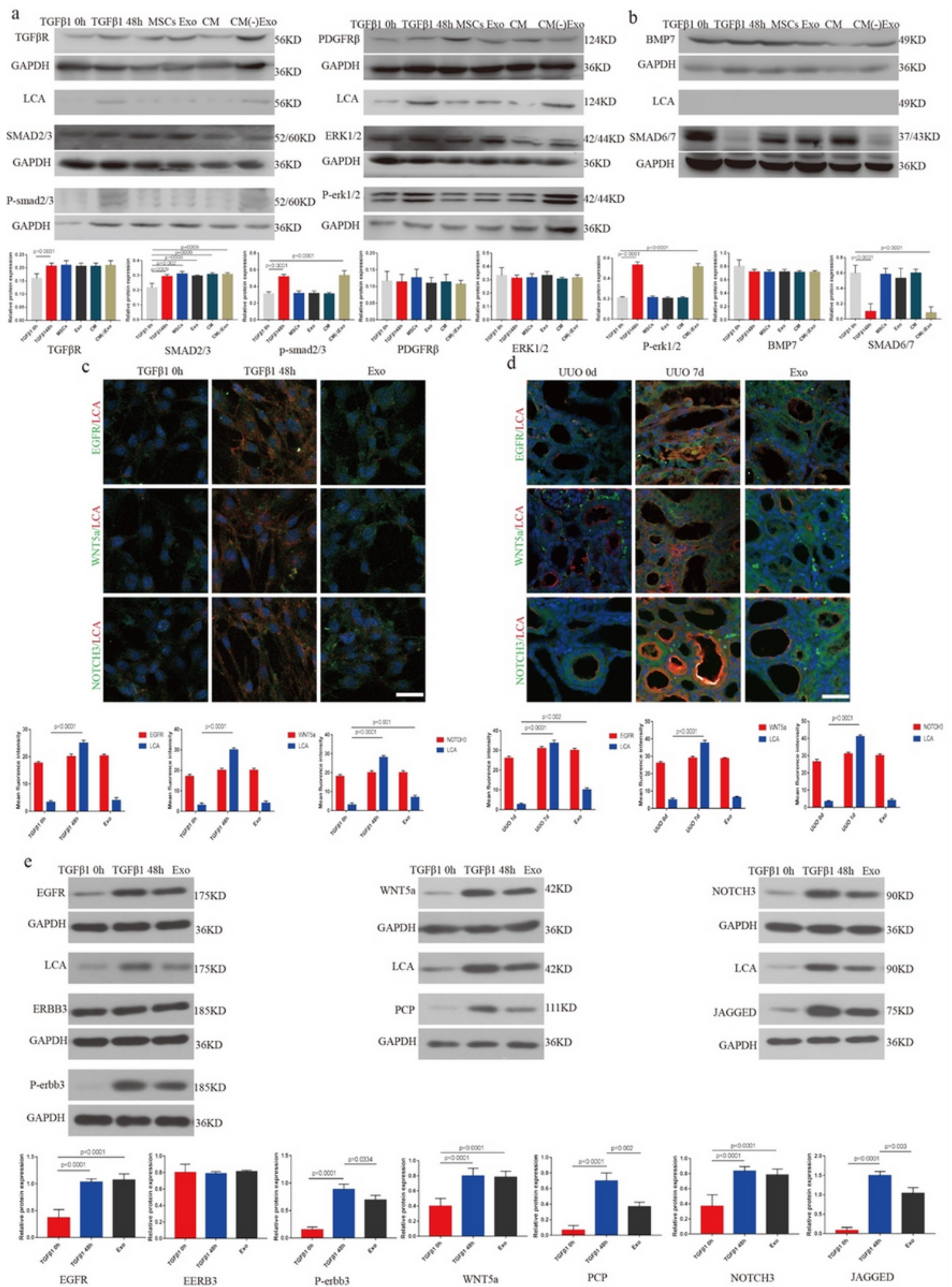

Figure 5 
Exosome-induced inhibition of CF inactivated multiple signaling pathways. a,b,e TGF $\beta R 1, P D G F R \beta, B M P$, EGFR, WNT5a, and NOTCH3 levels in total cell lysates were assessed by western blotting.

Immunoprecipitated TGF $\beta$ R1, PDGFR $\beta$, BMP, EGFR, WNT5a, and NOTCH3 proteins were analyzed by lectin blotting. TGF $\beta R 1$, PDGFR $\beta$, BMP, EGFR, WNT5a or NOTCH3 were immunoprecipitated from whole cell lysates with the corresponding antibodies, and the blots were probed with LCA. SMAD2/3, P-

SMAD2/3, ERK1/2, P-ERK1/2, SMAD6/7, ERBB3, P-ERBB3, PCP, and JAGGED levels were assessed using western blotting. Total cell lysates underwent immunoblotting. Representative data are shown. The bottom panel shows the quantification. Data are the mean $\pm S D(n=3)$. c Representative images of EGFR (green) and LCA (red) staining, WNT5a (green) and LCA (red) staining, and NOTCH3 (green) and LCA (red) staining in vitro. The bottom panel shows the quantification. Scale bar, $75 \mu \mathrm{m}$. Data are the mean \pm SD (n =3). $d$ Representative images of EGFR (green) and LCA (red) staining, WNT5a (green) and LCA (red) staining, and NOTCH3 (green) and LCA (red) staining in vivo. The bottom panel shows the quantification. Scale bar, $75 \mu \mathrm{m}$. Data are the mean \pm SD $(n=3)$. 


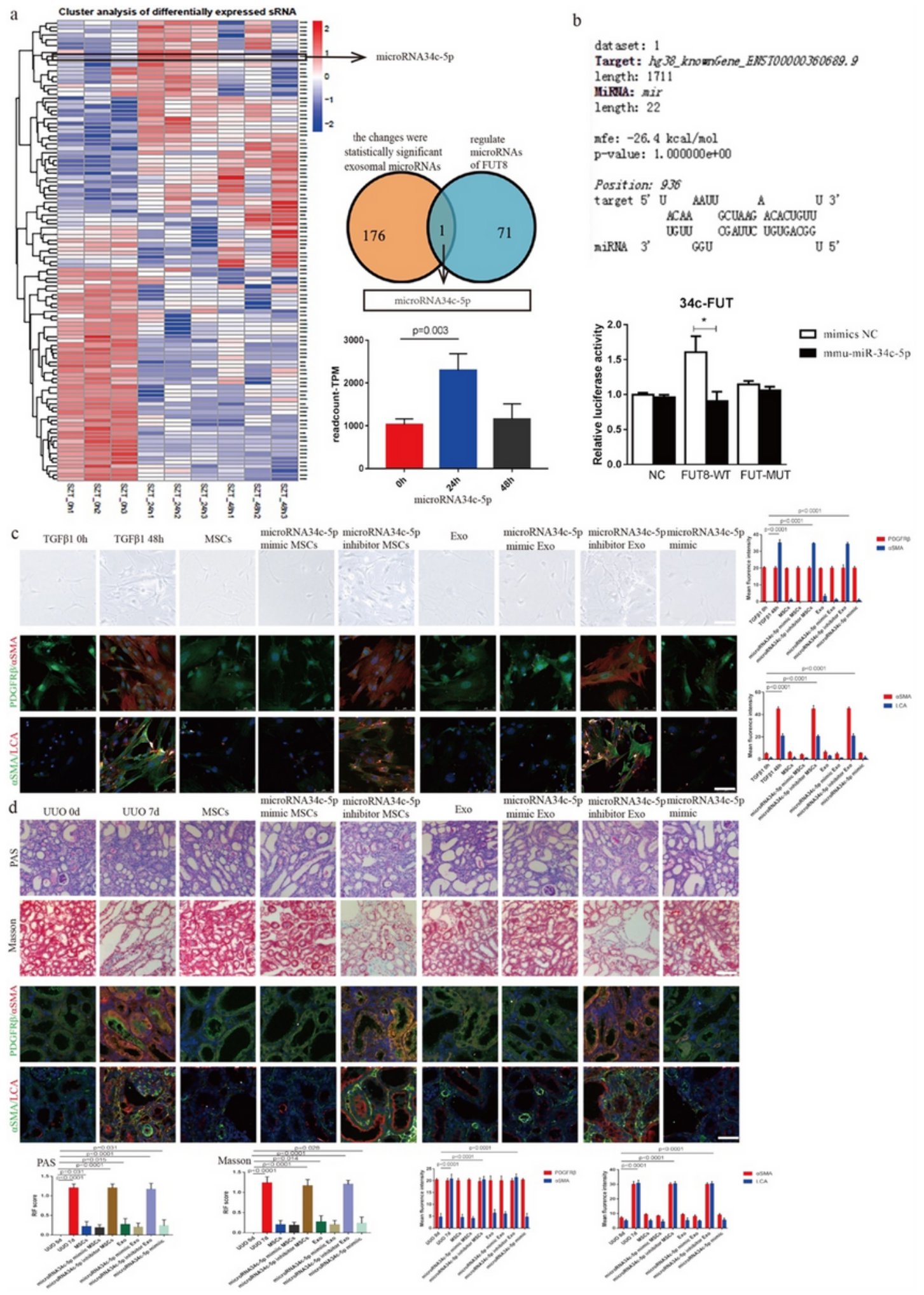

Figure 6

Exosomes downregulated CF by delivering miR-34c-5p during pericyte activation and renal fibrosis. a (Left) Heat map of exosomal miRNA and (right) histogram of miR-34c-5p at 0 h, 24 h, and 48 h. Data are the mean \pm SD $(n=3)$. b Luciferase reaction between FUT8 and miR-34c-5p. Data are the mean \pm SD $(n=$ 3). c Representative morphological alterations in pericytes. Scale bar, $200 \mu \mathrm{m}$. Also shown are representative images of PDGFRß (green) and a-SMA (red) staining, and a-SMA (green) and LCA (red) 
staining in vitro. The right panel shows the quantification. Scale bar, $75 \mu \mathrm{m}$. Data are the mean $\pm S D(n=$ 3). d Representative images of Masson's trichrome and PAS staining of UUO mouse kidney sections. Scale bar, $200 \mu \mathrm{m}$. Also shown are representative images of PDGFR $\beta$ (green) and a-SMA (red) staining, and a-SMA (green) and LCA (red) staining in vivo. The lower panel shows the quantification. Scale bar, 75 $\mu \mathrm{m}$. Data are the mean \pm SD $(n=3)$.

a
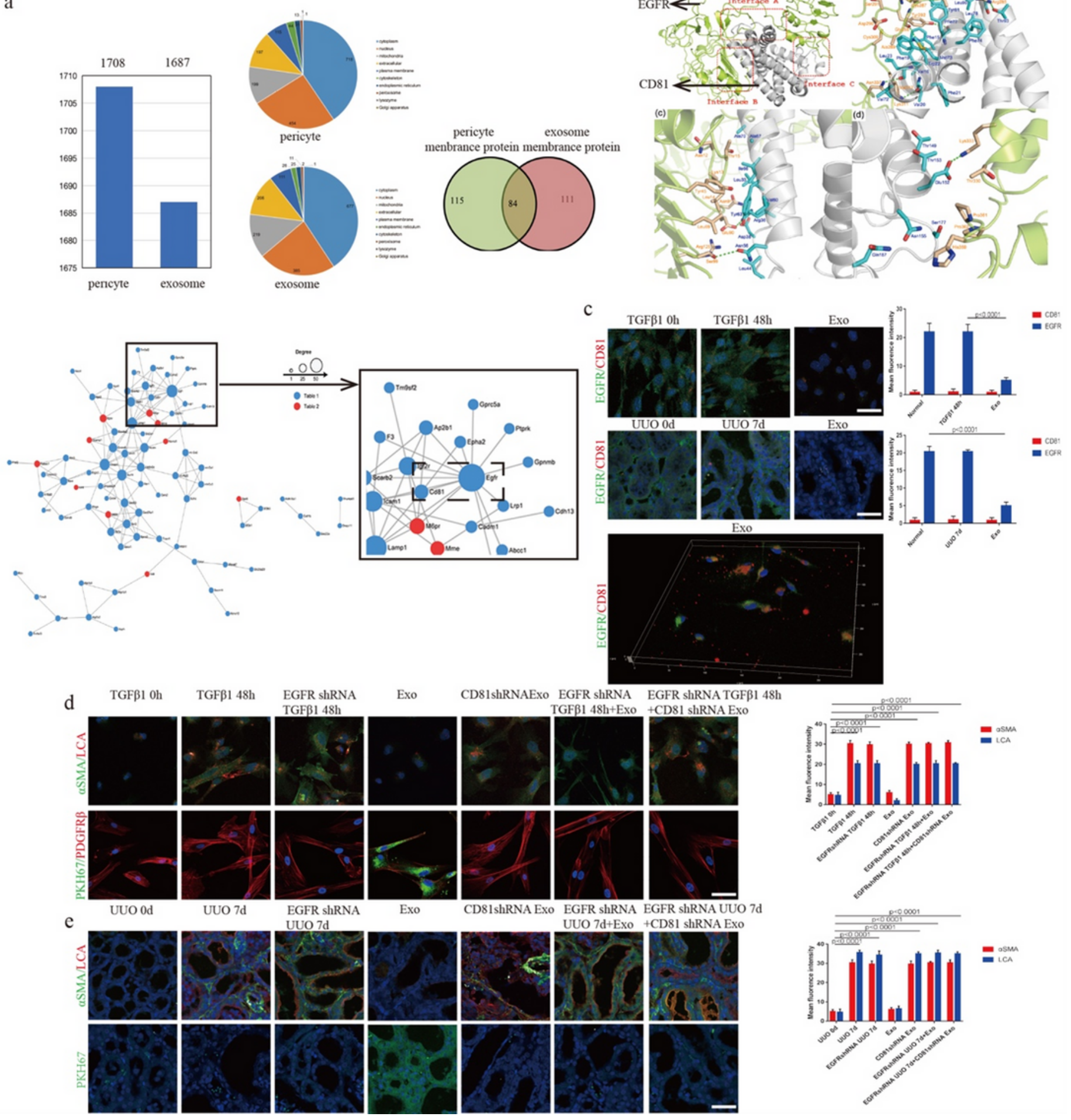

Figure 7 
CD81-EGFR complex formation aided miR-34c-5p entry into pericytes to downregulate CF. a (Top) Exosome and pericyte proteomics; (bottom) protein interaction network. b Computer simulation of CD81EGFR molecular docking. c Representative images of EGFR (green) and CD81 (red) staining both in vitro and in vivo. The right panel shows the quantification. Scale bar, $75 \mu \mathrm{m}$. Data are the mean $\pm S D(n=3)$. $d$ Representative images of a-SMA (green), LCA (red), and miR-34c-5p (fam) staining in vitro. The right panel shows the quantification. Scale bar, $75 \mu \mathrm{m}$. e Representative images of a-SMA (green), LCA (red), and miR-34c-5p (fam) staining in vivo. The right panel shows the quantification. Scale bar, $75 \mu \mathrm{m}$. Data are the mean $\pm \operatorname{SD}(n=3)$.

\section{Supplementary Files}

This is a list of supplementary files associated with this preprint. Click to download.

- supplementdataXuemeiHu.docx

- supplementaryinformation2020.11.05.docx 